OPEN ACCESS

Edited by:

Lei-Miao Yin,

Shanghai University of Traditional

Chinese Medicine, China

Reviewed by:

Soichiro Yamamura,

University of California, San Francisco,

United States

René-Marc Mège,

Centre National de la Recherche

Scientifique (CNRS), France

*Correspondence:

Klemens Rottner

klerottn@tu-braunschweig.de

Hans-Henning Arnold

h.arnold@tu-braunschweig.de

tPresent address:

Ines Lahmann,

Developmental Biology,

Max-Delbrück-Center for Molecular

Medicine, Berlin, Germany

Stefan A. Koestler,

Department of Physiology,

Development and Neuroscience, University of Cambridge, Cambridge, United Kingdom

Frieda Kage,

Department of Biochemistry and Cell Biology, Geisel School of Medicine at

Dartmouth, Hanover, $\mathrm{NH}$, United States

Georgi Dimchev,

Institute of Science and Technology Austria (IST Austria),

Klosterneuburg, Austria

Specialty section:

This article was submitted to

Cell Adhesion and Migration,

a section of the journal

Frontiers in Cell and Developmental

Biology

Received: 28 November 2020

Accepted: 07 January 2021

Published: 01 February 2021

\section{Induced Arp2/3 Complex Depletion Increases FMNL2/3 Formin Expression and Filopodia Formation}

\author{
Vanessa Dimchev ${ }^{1,2}$, Ines Lahmann ${ }^{1 \dagger}$, Stefan A. Koestler ${ }^{2 \dagger}$, Frieda Kage ${ }^{1,2+}$, \\ Georgi Dimchev ${ }^{1,2+}$, Anika Steffen ${ }^{2}$, Theresia E. B. Stradal ${ }^{2}$, Franz Vauti ${ }^{1}$, \\ Hans-Henning Arnold ${ }^{1 *}$ and Klemens Rottner ${ }^{1,2,3 *}$ \\ ${ }^{1}$ Zoological Institute, Technische Universität Braunschweig, Braunschweig, Germany, ${ }^{2}$ Department of Cell Biology, \\ Helmholtz Centre for Infection Research, Braunschweig, Germany, ${ }^{3}$ Braunschweig Integrated Centre of Systems Biology \\ (BRICS), Braunschweig, Germany
}

The Arp2/3 complex generates branched actin filament networks operating in cell edge protrusion and vesicle trafficking. Here we employ a conditional knockout mouse model permitting tissue- or cell-type specific deletion of the murine Actr3 gene (encoding Arp3). A functional Actr3 gene appeared essential for fibroblast viability and growth. Thus, we developed cell lines for exploring the consequences of acute, tamoxifen-induced Actr3 deletion causing near-complete loss of functional Arp2/3 complex expression as well as abolished lamellipodia formation and membrane ruffling, as expected. Interestingly, Arp3-depleted cells displayed enhanced rather than reduced cell spreading, employing numerous filopodia, and showed little defects in the rates of random cell migration. However, both exploration of new space by individual cells and collective migration were clearly compromised by the incapability to efficiently maintain directionality of migration, while the principal ability to chemotax was only moderately affected. Examination of actin remodeling at the cell periphery revealed reduced actin turnover rates in Arp2/3-deficient cells, clearly deviating from previous sequestration approaches. Most surprisingly, induced removal of Arp2/3 complexes reproducibly increased FMNL formin expression, which correlated with the explosive induction of filopodia formation. Our results thus highlight both direct and indirect effects of acute Arp2/3 complex removal on actin cytoskeleton regulation.

\footnotetext{
Keywords: F-actin branching, lamellipodium, filopodium, migration, chemotaxis, F-actin turnover, cell division, nuclear envelope breakdown
}

\section{INTRODUCTION}

Actin filament (F-actin) assembly and turnover in cells is tightly regulated by various actin monomer and filament binding proteins, with diverse functions. The generation of actin filaments de novo is referred to as nucleation, a complex process that received increasing attention over the past two decades (Campellone and Welch, 2010; Dominguez, 2010; Rottner et al., 2017). 
In living cells, spontaneous nucleation is likely prohibited by kinetic barriers, imposed for instance by actin monomer binding proteins, to avoid de-regulated, ectopic filament assembly. Cells overcome these barriers by different classes of filament assembly factors each operating at specific subcellular locations and time, allowing respective physiologic processes to occur. These F-actin assembly factors include the Arp $2 / 3$ complex plus various different activators, formins as well as the Spire/Cobl/Leiomodin type of actin binding proteins thought to enhance nucleation through simple actin monomer clustering (Chesarone and Goode, 2009; Qualmann and Kessels, 2009). Some members of these classes were also found to function cooperatively, as exemplified by Spire- or APCformin couples combining Spire/APC-mediated nucleation with formin-mediated elongation of generated actin filaments (Kerkhoff, 2011; Breitsprecher et al., 2012), although the precise modes of action of such cooperative complexes are still to be unraveled (Montaville et al., 2014). Other studies have implied antagonistic functions between actin assembly classes, e.g., between Arp2/3 complex and formins (Suarez et al., 2015; Kadzik et al., 2020), with the actin monomer-binding profilin operating as gatekeeper for Arp2/3-dependent vs. independent actin assembly (Rotty et al., 2015). This complexity thus requires development of individual and combined loss of function approaches and systematic and careful side-byside analysis.

The first and best characterized complex considered today as bona fide generator of rapidly-growing, barbed ends of actin filaments is the Arp2/3 complex (Goley and Welch, 2006), which amplifies actin filament assembly by branching daughter filaments off mother filaments that both continue to grow. In recent years, the Arp2/3 complex turned out to be of fundamental importance for numerous cellular processes, including actin-based cell edge protrusion during migration and various additional actin assembly processes at both plasma and intracellular membranes (Rotty et al., 2013; Molinie and Gautreau, 2018), as well as specific interactions of viral or bacterial pathogens with their host cells (Welch and Way, 2013). Interestingly, many of these processes, in particular those occurring at the plasma membrane, involve adaptive responses orchestrated by Arp2/3 complex to mechanical forces (Mueller et al., 2017; Akamatsu et al., 2020), as recently emphasized (Papalazarou and Machesky, 2020). Although the canonical complex is comprised of seven subunits throughout eukaryotic cell evolution, expression of functionally diverse or incomplete complexes-even within the same cell type-have also been described (Chorev et al., 2014; Abella et al., 2016). However,

\footnotetext{
Abbreviations: AOTF, Acousto-optical tunable filter; Arp2/3, actin-related protein 2/3; FMNL2, formin-like family member 2; FMNL3, formin-like family member 3; MEFs, mouse embryonic fibroblasts; Rho, Ras homolog gene family; Rac, ras-related C3 botulinum toxin substrate; RNAi, RNA interference; Scar, Suppressor of cAMP receptor; WAVE, WASP family verprolin-homologous; WRC, WAVE regulatory complex; VASP, Vasodilator-stimulated phosphoprotein; Ena/VASP, Enabled/vasodilator-stimulated phosphoprotein; EGFP, Enhanced Green Fluorescent Protein; FRAP, fluorescence recovery after photobleaching.
}

our understanding of their physiological relevance is still scarce (Pizarro-Cerda et al., 2017).

Canonical Arp2/3 complexes are activated to form actin filament branches by a family of so called nucleation promoting factors (NPFs), the name giving member of which, WASp, causes the rare, X-linked Wiskott-Aldrich-Syndrome, and contains the WCA-module (WH2 plus Connector plus Acidic) at its C-terminal end operating in actin binding (W) and Arp2/3 complex activation (CA). Although it comes in various forms, such as multiple copies of $\mathrm{W}$-domains, this module constitutes the minimal sequence necessary for Arp2/3 activation (Campellone and Welch, 2010), and is thus common to all class I NPFs. We generally agree today that the specificity of Arp2/3 complex functions in cells is largely mediated by temporal and spatial control through NPFs, potentially explaining how Arp2/3 complex activity can be precisely coordinated throughout cells and tissues. Activation of Arp $2 / 3$ complex in specialized protrusive structures termed lamellipodia, for instance, is generally thought to be mostly accomplished through the Rac GTPase-effector WAVE Regulatory Complex (WRC) (Krause and Gautreau, 2014; Alekhina et al., 2017; Schaks et al., 2018, 2019).

Various distinct experimental approaches have previously been utilized to interfere with Arp2/3 complex function directly, including classical approaches like RNA interference (Rogers et al., 2003; Di Nardo et al., 2005; Steffen et al., 2006; NicholsonDykstra and Higgs, 2008), inhibition by small molecules (Nolen et al., 2009) or even cytosolic Arp2/3 complex sequestration (Machesky and Insall, 1998; Hufner et al., 2001; Koestler et al., 2013). Yet, we are still falling short on tools for complete and reproducible elimination of Arp2/3 complex function in living cells, likely due to its vital role in cells (see below).

Aside from stable, simultaneous RNAi-mediated suppression of Arp2 and Arp-C2 (p34) in fibroblasts (Wu et al., 2012), genetic elimination of the Arp-C3 (p21) subunit allowed studying its consequences on ES cell-derived fibroblasts, albeit only for a limited number of cell divisions (Suraneni et al., 2012). These studies uncovered both, commonly shared and divergent views concerning Arp2/3 function in lamellipodia formation vs. migration efficiency or chemotaxis, which could not be resolved in respective follow-up studies (Wu et al., 2013; Suraneni et al., 2015). Here, we introduce the tamoxifen-inducible elimination of Arp2/3 function in several, independentlygenerated fibroblast cell lines, derived from a mouse mutant harboring a conditional Arp3 allele that can be removed by Cre-recombinase (Papalazarou et al., 2020). As opposed to previous studies employing Rac- or WRC-deficient cell lines (Steffen et al., 2013; Schaks et al., 2018) and in line with recent data analyzing dendritic cell migration in environments of varying complexity (Leithner et al., 2016), our results suggest that efficient, mesenchymal migration does not always involve lamellipodia, but that these structures become particularly relevant during processes requiring plasticity and establishment and/or maintenance of the direction of migration. Moreover, and distinct from previously published work on knockdown or knockout of the Arp2/3 complex (Suraneni et al., 2012; Wu et al., 2012), we confirm here, as already suggested by previous, 
independent studies (Steffen et al., 2013), that lamellipodia are dispensable for cell spreading. Finally, our analyses uncover that acute elimination of Arp2/3 complex function leads to upregulation of FMNL formin expression, which is largely responsible for the induced burst of filopodia formation.

\section{MATERIALS AND METHODS}

\section{Mice and Cell Lines}

Mice homozygous for the loxP-site flanked Act3r allele were generated as described previously (Papalazarou et al., 2020). For generation of fibroblast cell lines used in this study, E13.5-embryos lacking head and intestines were cut into pieces, digested with trypsin and single cells separated by thorough squeezing through a pipet tip before seeding into fibroblast growth medium. Cells were spontaneously immortalized through continuous passaging. Subsequently, cells were transfected with Cre-ERT2-plasmid, kindly provided by Pierre Chambon (Feil et al., 1997), leading to stable expression of the inducible Cre-recombinase variant in the cytoplasm of clonal cell lines selected by puromycin treatment $(2 \mu \mathrm{g} / \mathrm{ml})$. Tamoxifen treatment causes nuclear translocation of Crerecombinase (Metzger and Chambon, 2001), and subsequent disruption of floxed Act3r alleles. Three clones with comparable growth rates were selected for further analyses, termed clones 5, 7, and 19 (Lahmann, 2011). Following selection, cell lines were maintained in DMEM, $4.5 \mathrm{~g} / \mathrm{l}$ glucose (Gibco) supplemented with 10\% FCS (Sigma), $2 \mathrm{mM}$ L-glutamine (Gibco), $2 \mathrm{mM}$ penicillin/streptomycin (Gibco), $0.1 \mathrm{mM}$ nonessential amino acids (Gibco), $1 \mathrm{mM}$ sodium pyruvate (Gibco) and $5 \mu \mathrm{g} / \mathrm{ml}$ of puromycin (Sigma), unless indicated otherwise, and gene disruption routinely induced by treatment with (Z)-4Hydroxytamoxifen for $96 \mathrm{~h}$ (stock solution in DMSO/EtOH and used in a final concentration of $2 \mu \mathrm{g} / \mathrm{ml}$ ).

\section{Plasmids and Transfections}

Spontaneously immortalized fibroblast cell lines were transfected with Cre-ERT2 using Lipofectamine 2000 (ThermoFisher Scientific) following manufacturer's instructions. All other transfections were done with jetPRIME transfection reagent (Polyplus) according to manufacturer's protocol. For shRNAmediated knockdown of FMNL2 and FMNL3, corresponding psiRNA-h7SK-GFPzeo vectors (InvivoGen) harboring knockdown sequences 5'-GGAAGTCTGCGGATGAGAT AT-3' (FMNL2) and 5'-GGTGCAGATTCAAGCGTACCT-3' (FMNL3) were co-transfected in a 1:1 ratio, and subjected to FACS-sorting prior to tamoxifen/vehicle treatment. For FRAP (see below), EGFP-tagged human $\beta$-actin (Clontech, Mountain View, CA, USA) was used, as described previously (Steffen et al., 2013), and pRK5-myc-Rac1-L61 obtained from Laura Machesky (Cancer Research UK, Beatson Institute, UK). EGFP-tagged Arp3 was as described (Stradal et al., 2001), and for generation of EGFP-C2-Arp3B plasmid, the mRNA transcript variant 1 encoding murine actin-related protein 3B (Actr3b) (Jay et al., 2000) was synthesized (GenScript Biotech) and cloned into pEGFP-C2 vector (Clontech).

\section{Western Blotting and Intensity Quantifications}

Detergent-soluble cell extracts were routinely used for all Western blots, and prepared as described (Kage et al., 2017). Soluble protein concentrations were measured using Pierce ${ }^{\mathrm{TM}}$ BCA Protein Assay Kit (Thermo Scientific) and determined using a MRX microplate reader (Dynatech Laboratories). Western Blotting was performed according to standard procedures. Glyceraldehyde-3-Phosphate Dehydrogenase (GAPDH) levels were used as loading control. Primary antibodies were: GAPDH (clone 6C5, \#CB1001, Calbiochem, 1:10000 dilution), p16A (Arp-C5A, clone 323H3; homemade, undiluted hybridoma supernatant) (Olazabal et al., 2002), Arp2 (clone FMS69, Sigma; at a concentration of $1 \mu \mathrm{g} / \mathrm{ml}$ ), Arp3 (clone FMS338, Sigma, at a concentration of $1 \mu \mathrm{g} / \mathrm{ml}$ ), Arp3B (polyclonal rabbit Anti-ACTR3B, \#ARP57420_P050, Aviva Systems Biology, at a concentration of $1 \mu \mathrm{g} / \mathrm{ml}$ ), p34 (Arp-C2, \#ab205718, Abcam, 1:1,000 dilution), FMNL2/3 (\#ab57963, Abcam, 1:1,000 dilution), Rac1/3 (clone 23A8, Upstate, 1:2,000 dilution), $B$-actin (AC15, \#A1978, Sigma, 1:10,000 dilution), Sra-1 (rabbit polyclonal 4955B, 1:2,000 dilution) and Nap1 (rabbit polyclonal 4953B, 1:5,000 dilution) (Steffen et al., 2004). Polyclonal rabbit antisera to Abi-1 (1:2,000 dilution) and Arp-C1 (p40, 1:500 dilution) were raised (by Eurogentec Deutschland GmbH, Köln, Germany) against the synthetic peptides PPVDYEDEEAAVVQYNDPY ADGDPAWAPKNYI derived from the human Abi-1 sequence and TARERFQNLDKKASSEGGTAAG derived from the human Arp-C1B sequence, respectively, and affinity-purified using corresponding peptides immobilized on $\mathrm{CNBr}$-sepharose $4 \mathrm{~B}$ (Amersham Biosciences, Sweden). Specificity of the antisera was confirmed by Western blot detection of the endogenous and ectopically expressed EGFP-tagged proteins. As secondary antibodies, we used peroxidase-coupled anti-mouse IgG (\#111035-062, 1:10,000 dilution) and anti-rabbit IgG (\#111-035-045, 1: 10,000 dilution), which were purchased from Dianova. Protein bands were visualized using chemiluminescent, peroxidase substrate (Lumi-Light Western Blotting Substrate \#12015200001, Roche) and an ECL Chemocam Imager (Intas). Quantifications of protein levels from exposed Western blot membranes were done as described (Kage et al., 2017).

\section{Immunolabelings}

For immunolabellings, MEFs were seeded subconfluently o/n (unless indicated otherwise) onto coverslips acid-washed and coated with human fibronectin $(25 \mu \mathrm{g} / \mathrm{ml}$, \#11051407001, Roche), as described (Dimchev and Rottner, 2018). For spreading assays, fibroblasts were fixed after different times as indicated in Figure 4, Supplementary Figures 7, 8. For timepoint 0, coverslips were coated with poly-L-lysin (PLL, Sigma, $0.1 \mathrm{mg} / \mathrm{ml}$ ) followed by three PBS-washes, and fibroblasts centrifuged onto coverslips for $2 \mathrm{~min}$ at $1,200 \mathrm{rpm}$ prior to fixation.

After brief washing with PBS, cells were routinely fixed with 4\% paraformaldehyde (PFA) in PBS for $20 \mathrm{~min}$, and permeabilized with $0.1 \%$ Triton X-100 (Sigma) in PBS for $60 \mathrm{~s}$, unless indicated otherwise. For phalloidin stainings, glutaraldehyde (EM Grade) was added to fixative (0.25\%). For 
vinculin stainings, fibroblasts were permeabilized with $0.3 \%$ Triton X-100/PBS for $1 \mathrm{~min}$ prior to fixation with 4\% PFA/PBS for $20 \mathrm{~min}$. All solutions were pre-warmed to $37^{\circ} \mathrm{C}$.

Focal adhesions were stained with monoclonal antivinculin antibody (\#V9131, Sigma, 1:250 dilution), centrosomes with polyclonal anti-gamma tubulin antibody (PA1-28042, ThermoFisher, 1:1,500 dilution), and WRC at the cell periphery (Supplementary Figure 3) using monoclonal anti-Abi-1 (supernatant of clone 8.3, 1:50 dilution, kindly provided by Giorgio Scita, IFOM Milano). Secondary reagents were Alexa Fluor 594-coupled anti-mouse IgG (\#A11032, Invitrogen, 1:200 dilution) and Alexa Fluor 488-coupled anti-rabbit IgG (\#A11034, Invitrogen, 1:400 dilution). Phalloidins were either ATTO488-coupled (\#AD488-81, ATTO-TEC, 1:200 dilution), ATTO594-coupled (\#AD594-81, ATTO-TEC, 1:200 dilution) or ATTO390-coupled (\#AD390-81, ATTO-TEC, 1:200 dilution), dependent on experiment and the combinations with antibodies. Nuclei were routinely stained with DAPI, already present in the mounting medium (ProLong Gold, \#P36941, Invitrogen) or, for SIM-experiments, with TO-PRO-3 (\#T3605, ThermoFisher, 1:1,000 dilution).

\section{Conventional Fluorescence and Superresolution Microscopy and FRAP}

Images of immunolabelings were acquired on an inverted Axiovert 100TV epifluorescence microscope (Zeiss) using a 40x/1.3 NA Plan-Neofluar oil immersion objective. The microscope was equipped with an HXP 120 light source for widefield fluorescence illumination, and a Coolsnap-HQ2 camera (Photometrics) as well as electronic shutters (Uniblitz Corporate) driven by VisiView software (Visitron Systems GmbH, Puchheim, Germany).

Structured illumination microscopy (SIM) data were captured using a CFI Apochromat TIRF 100x/1.49 NA oil immersion objective (Nikon) on a Nikon SIM-E superresolution microscope equipped with a LU-N3-SIM 488/561/640 laser unit mounted on a Nikon Ti eclipse. Image acquisition was enabled using a NIS-Elements software (Nikon)-driven N-SIM motorized quad band filter combined with N-SIM 488 and 561 bandpass emission filters and a Hamamatsu Orca flash 4.0 LT camera.

Fluorescence recovery after photobleaching (FRAP) experiments were performed as described (Dimchev and Rottner, 2018). Specifically here, vehicle control or tamoxifentreated MEFs were transfected with EGFP-ß-actin followed by $\mathrm{o} / \mathrm{n}$ seeding onto fibronectin-coated coverslips, and subjected to FRAP experiments the following day.

\section{Random Migration, Chemotaxis, and Wound Healing Assays}

For random migration assays, $\mathrm{DMSO} / \mathrm{EtOH}$ - and respective tamoxifen-treated fibroblast clones were seeded subconfluently into $\mu$-Slide $4 \mathrm{Well} \mathrm{Ph}+$ Glass Bottom chambers (Ibidi) coated with human fibronectin $(25 \mu \mathrm{g} / \mathrm{ml}) 6 \mathrm{~h}$ prior to time-lapse, phase-contrast microscopy.

Prior to chemotaxis assays, respective cell populations were starved overnight in DMEM and seeded subconfluently in non-coated $\mu$-slide chemotaxis ${ }^{2 \mathrm{D}}$ chambers (Ibidi) $3 \mathrm{~h}$ prior to chemotaxis assays. Chemotaxis experiments were performed according to manufacturer's instructions. In brief, both chemoattractant chambers were filled with DMEM before adding $1 / 4$ of total chamber volume of a $4 \mathrm{x}$ chemoattractant stock solution to the upper chamber $\left(\mathrm{C}_{100}\right)$ in order to obtain a final gradient concentration of $2.5 \%$ FCS and $100 \mathrm{ng} / \mathrm{ml} \mathrm{HGF}$ (\#H9661, Sigma) in DMEM.

Wound healing assays were performed on cell monolayers grown on fibronectin-coated glass bottom dishes upon removal of standardized, wound-creating silicone culture-inserts (Ibidi) or wounding with a disposable pipet tip.

Time-lapse microscopy data were generated on an inverted Axio Observer (Zeiss) utilizing a 10x/0.3NA Plan-Neofluar air objective for random migration and chemotaxis or a 20x/0.4NA LD Achroplan objective for wound-healing assays. Cells were kept at $37^{\circ} \mathrm{C}$ and a $\mathrm{CO}_{2}$ concentration of 7.5 using an incubation chamber (Incubator XL multi S1, Zeiss) connected to a heating and $\mathrm{CO}_{2}$ control unit (PeCon), and maintained in full medium (except for chemotaxis assays). Random migration and chemotaxis were acquired over a timeperiod of $20 \mathrm{~h}$ and at a frame rate of $4 / \mathrm{h}$, whereas woundhealing data were also recorded for up to $20 \mathrm{~h}$ with a frame rate of $12 / \mathrm{h}$.

\section{Data Analysis and Processing}

Numbers of cells with lamellipodia and numbers of filopodia or concave edges per cell were counted manually from phalloidin images. DAPI and gamma tubulin stainings were employed to manually quantify the number of nuclei and centrosomes, respectively. The length of concave edges was also assessed manually but aided by ImageJ Software. Analysis of spreading behavior and quantification of adhesion numbers and sizes were performed as described in Steffen et al. (2013) and Kage et al. (2017), respectively.

Image plugins "manual tracking" and "chemotaxis tool" were used to analyze random migration and chemotaxis. DiPer software (Gorelik and Gautreau, 2014) was utilized to display the individual cell tracks in Supplementary Figure 9 and to perform mean square displacement (Figure 6B) as well as direction autocorrelation analysis (Figure 6C) from random migration data.

Collective migration speed of control and tamoxifen-treated fibroblast populations in wound healing assays was assessed as follows: In order to obtain the distance migrated by the cell monolayer, the area engaged was measured at the beginning and after $720 \mathrm{~min}(12 \mathrm{~h})$ of the wound closure process, as described previously (Steffen et al., 2013), and divided by the width of the camera chip to normalize for employment of distinct setups in the course of time. Migration distances from individual experiments were then divided by time to obtain migration velocities, averaged and displayed as bar charts.

Data obtained from FRAP experiments were analyzed as follows: Half-times of recovery were derived from fluorescence recovery curves as previously described (Koestler et al., 2013; Steffen et al., 2013; Dimchev and Rottner, 2018). Due to the comparably narrow lamellipodia formed by these fibroblasts and 
in control conditions, fluorescence intensities within a region of $1 \mu \mathrm{m}$ at the lamellipodium front of control or the corresponding cell periphery of tamoxifen-treated cells were measured using MetaMorph Software (Molecular Devices). Fitted curves were generated in Sigma plot 12.0 (Systat Software) by applying dynamic curve fits for exponential rise to maximum using $f(x)=$ $y_{0}+a *\left(1-e^{-b * x}\right)$.

For calculation of the treadmilling factors (TMF) (Lai et al., 2008), the $1 \mu \mathrm{m}$ region of intensity measurements described above was subdivided into two regions of identical depths $(0.5 \mu \mathrm{m}$ each), referred to as front and back (see Figure 3 and Supplementary Figure 6). Two separate recovery curves for front and back regions were generated and, in case of control cells, fitted employing a dynamic curve fit for exponential rise to maximum: $f(x)=y_{0}+a *\left(1-e^{-b * x}\right)$ for data derived from the front part, and following a sigmoidal curve: $f(x)=$ $y_{0}+\frac{a}{1+e^{-\frac{x-x_{0}}{b}}}$ for data derived from the rear part. In contrast, recovery curves from tamoxifen-treated cells were best fitted using equation $f(x)=y_{0}+a *\left(1-e^{-b * x}\right)$ for both front and back region. The treadmilling factor was derived from these curves as described (Lai et al., 2008).

MetaMorph Software was used to adjust brightness and contrast levels of all images assembled into final figure panels employing Adobe Photoshop CS5 software. Raw data were analyzed and processed in ImageJ, MetaMorph, Excel 2016 (Microsoft) and Sigma plot 12.0.

\section{Statistics}

Statistical significance was assessed using $t$-test or nonparametric Mann-Whitney rank sum test (Sigma plot 12.0) if datasets were not normally distributed (according to AndersonDarling normality test), as indicated in individual figure legends. Statistical significance is expressed by the number of asterisks, with * corresponding to a $\mathrm{p}$ value smaller than 0.05 , and ${ }^{* *}$ and *** corresponding to $p<0.01$ and $p<0.001$, respectively.

\section{RESULTS AND DISCUSSION}

\section{Acute Removal of Arp3 Suppresses the Arp2/3 Complex and Lamellipodia Formation}

In our initial efforts to generate fibroblast cell lines completely devoid of the Arp2/3 complex subunit Arp3, MEFs homozygous for the conditional Actr3 allele described previously (Lahmann, 2011; Papalazarou et al., 2020) were both SV40-LT-antigen- or spontaneously immortalized, and subjected to clone isolation and analysis following expression of Cre-recombinase. However, in spite of numerous attempts to isolate homozygously-deleted Actr3 clones, as successfully achieved for instance in case of the Rho-GTPase Racl (Steffen et al., 2013), we were unable to obtain viable Actr3 null MEFs (data not shown and see below). This indicated that Actr3 is essential for cell viability and growth, as suggested previously for Arp-C2 and Arp2 in fibroblasts, at least in the presence of tumor suppressors
(Ink4a/Arf) (Wu et al., 2012), and unlike the recent description of Arp2 null HL-60 promyeloblasts (Graziano et al., 2019). We thus turned to exploring the effects of acute Actr3 disruption, which was achieved in spontaneously-immortalized cell lines stably expressing tamoxifen-inducible Cre recombinase. Three independent cell clones were isolated (termed Arp3.5, 7, and 19) and analyzed separately for Arp3 expression after 3, 4, and 5 full days of treatment with tamoxifen or DMSO/EtOH as vehicle control (Figure 1A). This allowed determining 4 days $(96 \mathrm{~h}$ ) of tamoxifen treatment as optimal compromise between Arp3 elimination and cell viability (condition boxed red in Figure 1A). The severe reduction of Arp3 expression after $96 \mathrm{~h}$ correlated well with reduced expression of other Arp2/3 complex subunits tested, i.e., Arp2, Arp-C1, Arp-C2, and Arp-C5 in all three clones (Figure 1B). This confirmed the view that Arp2/3 complex subunits are largely dependent on each other in cells, as observed previously at least for functional interference with Arp3 (Di Nardo et al., 2005; Steffen et al., 2006) and Arp-C3 (Suraneni et al., 2012), and due perhaps to instability or active degradation of most versions of partial complexes.

To start examining the consequences of acute Arp3 suppression, we quantified the frequency of lamellipodium formation, one of the most prominent structures considered to require functional Arp2/3 complexes (Suraneni et al., 2012, 2015; Wu et al., 2012; King et al., 2016). Not unexpectedly, although at variance to the conclusions drawn from one previous Arp3 RNAi study (Di Nardo et al., 2005), lamellipodia formation was strongly suppressed upon Arp3 deletion in all three clonal fibroblast cell lines growing on fibronectin (Figures 2A,B for quantification). Notably, aside from the elimination of lamellipodia, cells were also significantly increased in size under these conditions (note size bars in Figure 2A), suggesting that loss of lamellipodia does not necessarily interfere with the expansion of cell area on 2D-surfaces (see also below). Canonical lamellipodia formation is well established today to require Rac GTPase signaling to WRC-mediated Arp2/3 complex activation (Steffen et al., 2013; Schaks et al., 2018; Rottner and Schaks, 2019). So to confirm that loss of lamellipodia in these conditions was due to lack of Arp2/3 complex expression, and not due to loss of upstream components of the signaling pathway, several control experiments were performed. Ninety six hours of tamoxifen treatment did not reduce Rac expression in any of the cell lines employed, as shown using an antibody cross-reactive with Racl and-3 (Steffen et al., 2013); instead, a trend toward increased Rac1/3 expression was observed, statistically significant at least in two out of three cell lines (Supplementary Figure 1). Interestingly, the Sra-1/PIR121 and Nap1 subunits of the Rac downstream effector WRC were virtually unchanged in expression, whereas the Abl interactor Abi-1 was reduced to about half of controls, for unknown reasons (Supplementary Figure 2). However, immunolabelling experiments confirmed the presence of Abi-1 at the cell edges of both vehicle control and tamoxifen-treated cells (Supplementary Figure 3), strongly suggesting that the loss of lamellipodia in tamoxifen-treated cells was not caused by the elimination of Abi-1 or other WRC subunits. Finally, the loss of actin filament-rich lamellipodia also did 

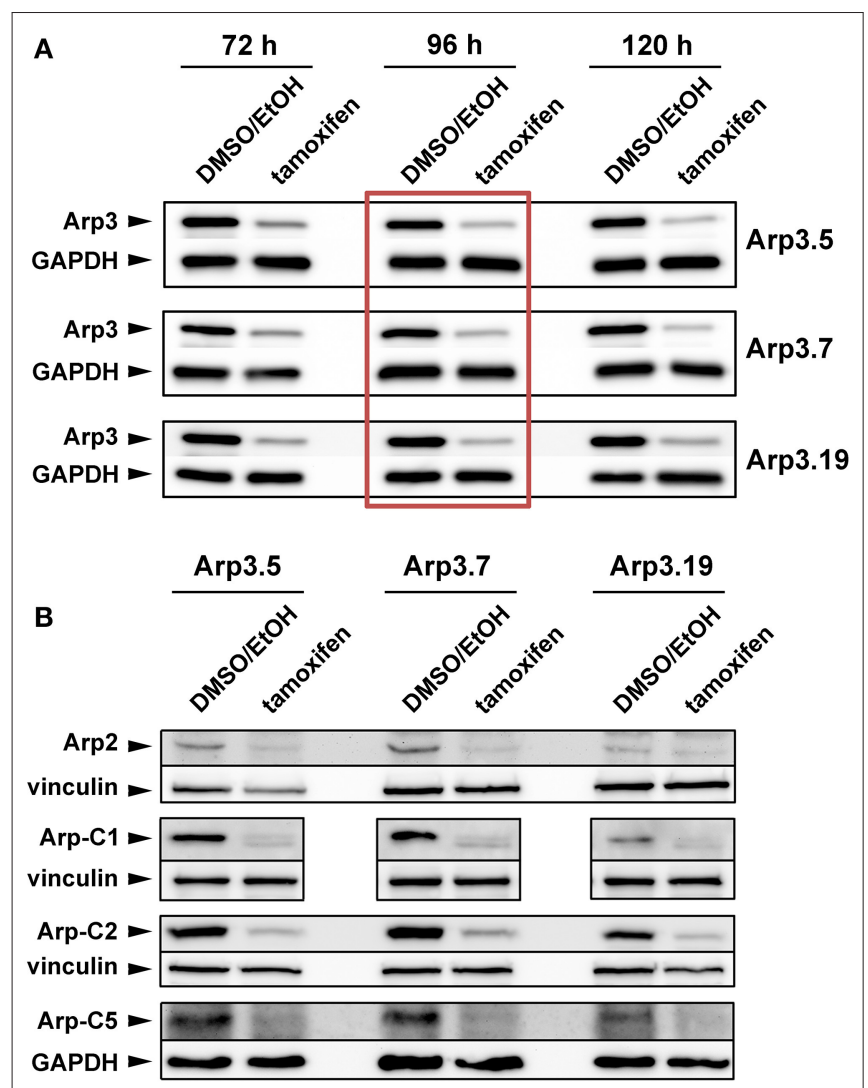

FIGURE 1 | Tamoxifen treatment suppresses expression of Arp2/3 complex subunits. (A,B) Western blot analysis of Arp2/3 complex subunit levels in individual $A c t r 3^{f / / f l}$ clones after tamoxifen treatment $(72,96$, or $120 \mathrm{~h}$ ) or DMSO/EtOH used as vehicle control. GAPDH and vinculin served as loading controls, dependent on the molecular weight of explored Arp2/3 complex subunit. (A) Although the extent of Arp3 protein decrease correlated with prolonged tamoxifen treatment, a treatment of $96 \mathrm{~h}$ (red box) was determined as best compromise between efficient protein run down and cell viability, which was significantly compromised upon $120 \mathrm{~h}$ (not shown). The $96 \mathrm{~h}$-time point was thus used for all future experiments. (B) Tamoxifen-induced removal of Arp3 (96h) concomitantly reduced all additional Arp2/3 complex subunits tested, as indicated.

not correlate with a reduction in actin expression, at least as assessed for the prominent, cytoplasmic $\beta$-actin isoform (Supplementary Figure 4). All this suggested that the loss of lamellipodia upon $96 \mathrm{~h}$ tamoxifen treatment was caused by suppression of Arp3 and consequently Arp2/3 complex. As final confirmation of this, we found transfection with EGFP-tagged Arp3 to rescue lamellipodia formation in tamoxifen-treated cells in a fashion that was similar in extent to transfection with the related Arp3B (Supplementary Figure 5). Notably, using antibodies that could clearly distinguish Arp3 from Arp3B encoded in murine cells by the Actr3b gene, we found that Arp3B was undetectable at the protein level with and without Actr3 deletion (Supplementary Figure 5A). This confirmed that as opposed to an exciting study posted recently (Galloni et al., 2020), Arp3B is not relevant for the cell type and experiments used here, and was consistent with the nearly complete loss of lamellipodia formation upon induced, sole Actr3 deletion (Figure 1 and Supplementary Figure 5B).

\section{Arp3 Depletion Abrogates Both Peripheral Actin Network Treadmilling and F-actin Turnover}

Lamellipodia are driven by continuous activation and incorporation of Arp2/3 complex into the actin filament network polymerizing at the edge (Lai et al., 2008). This actin network treadmilling behavior in the lamellipodium was previously disrupted in our lab by the alternative approach of acute inhibition of Arp2/3 complex via its sequestration by microinjection of Scar/WAVE1-WCA (Koestler et al., 2013). In those WCA injection experiments, the network treadmilling behavior was abolished, but the rate of actin turnover was largely maintained (Koestler et al., 2013), quite distinct from genetic removal of Rac, which strongly reduced F-actin turnover at the cell periphery as well (Steffen et al., 2013). Here we demonstrate a nearly complete elimination of actin network treadmilling at the periphery of cells upon suppression of Arp3 expression (Figures 3A,B), but also a severe reduction of actin network turnover (Figure 3C for representative clone 19) to more than double of the fluorescence half time of recovery rate in the $\mathrm{KO}$ as compared to control. Essentially the same pattern was observed when cells were transfected with myc-tagged, constitutively active Rac1 (Supplementary Figure 6), which entirely failed to stimulate lamellipodia formation in Arp3-deficient cells. Highly comparable results were obtained with clones 5 and 7 both with and without stimulation by constitutively active Rac1 (data not shown). Although the differential response of actin turnover at the cell periphery upon Rac1-KO and Arp2/3 complex sequestration was noted and discussed previously, it remained unclear whether it derived from choice of target protein or experimental approach employed (Steffen et al., 2014). The data shown here establish aforementioned differential response not to derive from choice of protein. Although we cannot exclude that the differences in effects observed upon Arp2/3 complex sequestration (Koestler et al., 2013) vs. depletion may at least in part reflect the differential timing in development of the phenotype (seconds and minutes in case of sequestration vs. hours and days in case of induced depletion), the data provided here confirm that sustained interference with Rac signaling and downstream lamellipodial Arp2/3 complex activation abrogates both actin network treadmilling and F-actin turnover.

\section{Arp2/3 Complex Is Not Required for Fibroblast Cell Spreading}

Previous studies unanimously concluded that Arp2/3 complex and Arp2/3 complex-dependent lamellipodia are relevant for cell spreading (Suraneni et al., 2012; Wu et al., 2012). However, this view was challenged by our data on Rac-deficient fibroblasts that are also completely devoid of lamellipodia (Steffen et al., 2013), indicating that previous observations derived from specific, Arp2/3 abrogation-dependent roles in spreading rather than a general, lamellipodia-dependent effect. Interestingly, acute 

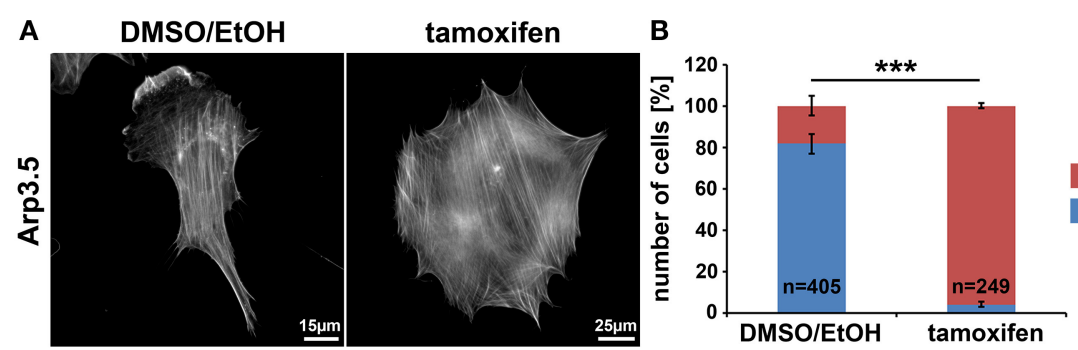

no lamellipodia

lamellipodia
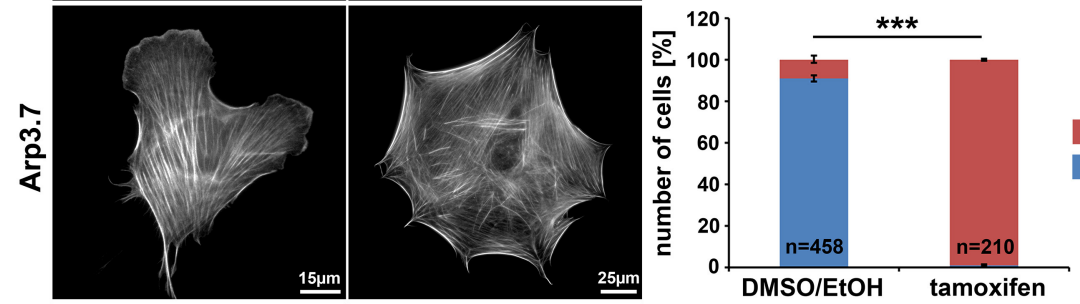

no lamellipodia

lamellipodia
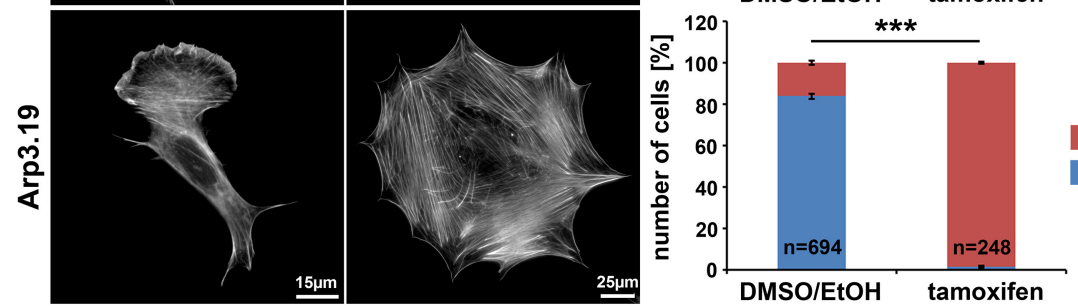

no lamellipodia

lamellipodia

FIGURE 2 | Arp3-depleted MEFs are devoid of lamellipodia. (A) Phalloidin-stained examples of distinct Actr3 ${ }^{f l / f l}$ clones (Arp3.5, Arp3.7, and Arp3.19) stably expressing tamoxifen-inducible Cre recombinase, with (tamoxifen) or without (DMSO/EtOH) tamoxifen induction (96 h) causing disruption of the Actr3 gene. Tamoxifen treatment causes near to complete elimination of lamellipodia in representative cells shown. Scale bars as indicated in individual panels. Note the strong increase of spread cell area upon Arp3 disruption. (B) Quantification of lamellipodia formation efficiency upon treatments as shown in $\mathbf{A}$. Data are arithmetic means and standard errors of means from three independent experiments; $n=$ total number of cells analyzed; data were statistically compared using two-sided, two-sample $t$-test ( ${ }^{\star \star \star} p<0.001$ ).

suppression of Arp3 expression dramatically increased cell size, but did not reduce spreading efficiency, as assessed from comparing the cell area covering the substratum surface at various time points after seeding (Figures 4A,B). Even if we normalized to the dramatic increase in cell size after $96 \mathrm{~h}$ of tamoxifen treatment, no substantial relative difference in spreading was recorded at any time point after seeding, neither for clone 19 (Figure 4C) nor clone 7 (Supplementary Figure 7). Only clone 5 slightly lagged behind at early spreading times if considering differences in cell size (Supplementary Figure 8C), but this difference was eliminated when data from all three clones were pooled (Supplementary Figure 8D). This strongly suggests that clonal variation in this case might explain the observation rather than a genotype-specific effect. We conclude that by and large, the loss of Arp3 and thus Arp2/3 function in actin remodeling and lamellipodia formation can be compensated for by cells in order to explore new space during spreading. As deduced from earlier research, this process likely involved filopodia formation (Wu et al., 2012; Steffen et al., 2013; Suraneni et al., 2015). To confirm this, we performed phase contrast video microscopy of cells immediately after seeding on fibronectin (Supplementary Video 1). Whereas wildtype cells spread using both, short filopodia and lamellipodia, Arp3-deficient cells initially protruded numerous, prominent and long filopodia, which then served as tracks for the advancement of cytoplasm in between them (Supplementary Video 1). Together, these data clearly suggest that at least in fibroblasts, cell spreading can be entirely uncoupled from both lamellipodia formation and Arp2/3 complex function.

\section{Arp2/3 Complex Promotes Directional Migration, but Is Not Essential for Chemotaxis}

Arp2/3 complex is commonly agreed to be crucial for haptotaxis in which cells follow gradients of ECM components or surfaceattached chemoattractants (King et al., 2016; Swaney and Li, 2016), but whether or not Arp2/3-depleted cells display a cellautonomous defect in chemotaxis has remained controversial (Suraneni et al., 2012, 2015; Wu et al., 2012, 2013). In classical wound healing experiments, our three cell lines displayed a significant defect in collective wound closure velocity upon acute Arp3 depletion as compared to EtOH-treated controls (Figure 5 and Supplementary Video 2). These data were highly reminiscent of previous data obtained with Arp-C3-KO, ES cell-derived fibroblastoid cells (Suraneni et al., 2012). However, this could not simply be explained by a general reduction of migration efficiency, as cell speed was not or only moderately reduced in random migration assays performed with clones 5 and 7/19, respectively (Figure 6A). Nevertheless, a more detailed analysis of random migration tracks from the same experiments (Supplementary Figure 9) and of mean square displacement revealed that in spite of comparable migration rates (Figure 6A), 
A

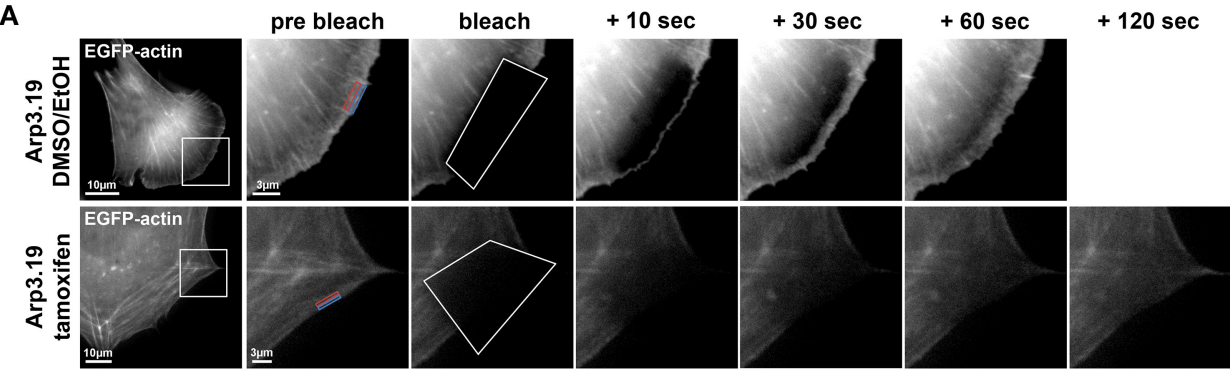

B

Fitted data (calculation of treadmilling factor)
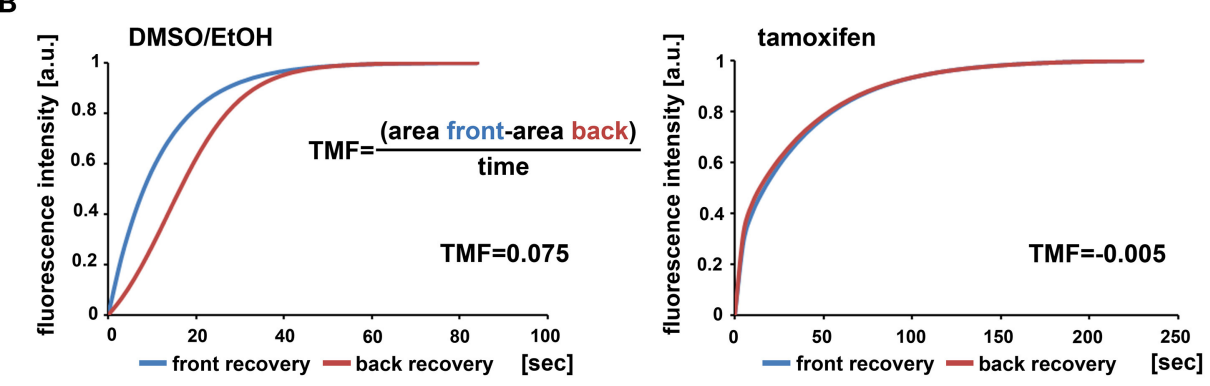

C

Data normalised to pre-bleach
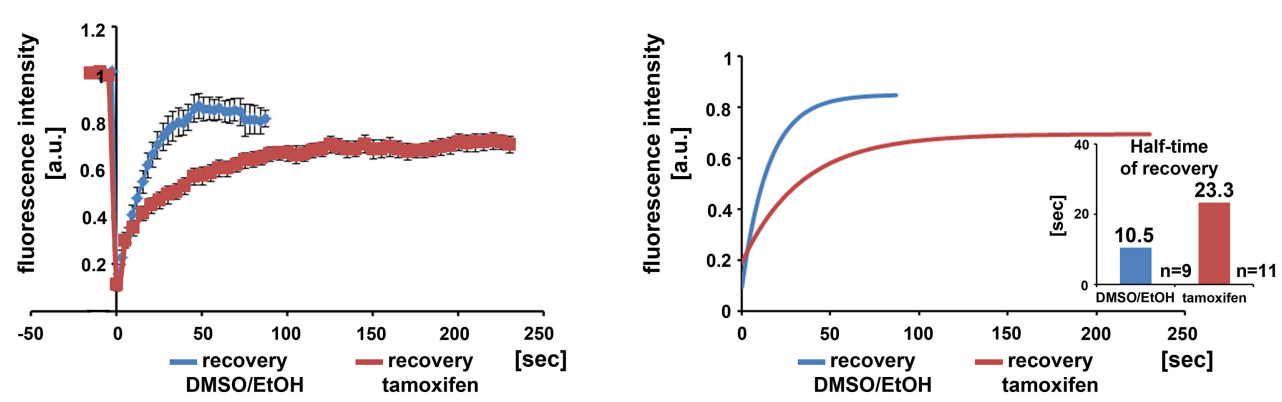

FIGURE 3 | Loss of the Arp2/3 complex interferes with treadmilling and F-actin turnover at the cell periphery. (A) Time-lapse images of representative FRAP experiments in Actr $3^{t / f l}$ cells with or without tamoxifen treatment (clone Arp3.19) expressing EGFP-actin. Regions of interest (ROI) are marked with white rectangles in overview images on the left. Different time points of enlarged ROI on right-hand panels display fluorescence signals of EGFP-actin immediately pre and after bleach as well as at different time points of fluorescence recovery. White polygons highlight photobleached regions at the cell periphery. Changes in signal intensities over time for calculations of treadmilling factors (TMF) were quantified within a region of $0.5 \mu \mathrm{m}$ at the front part (blue rectangle) as well as back part (red rectangle) of the lamellipodium in the Actr $3^{f / f / f l}$ cell or the corresponding periphery of the cell following Arp3 depletion. (B) Average fitted recovery curves of front (blue) and back (red) regions of Arp3-expressing (left) and Arp3-depleted cells (right) used to calculate the TMF utilizing the illustrated equation. Data were collected from 9 control and 11 tamoxifen-treated cells acquired in three independent experimental days. The treadmilling factor is defined as average difference in fluorescence recovery in the distal vs. proximal half of the lamellipodium. Note that this difference is absent in tamoxifen-treated cells, demonstrating that presence of a functional Arp2/3 complex is essential for bona fide treadmilling at the cell periphery. (C) EGFP-actin FRAP recovery curves within a $1 \mu \mathrm{m}$ deep peripheral region of DMSO/EtOH (blue) vs. tamoxifen-treated (red) Arp3.19 cells. Analysis was performed on the same time-lapse movies as used for the analysis in (B); data are arithmetic means and SEMs from movies the pre-bleach intensities of which were normalized to 1. Right panel shows fitted curves derived from raw data depicted on the left. Bar chart displays half times of fluorescent recovery as calculated from fitted curves. Note the marked decrease of F-actin turnover (increase of half-time of EGFP-actin fluorescence recovery) upon Arp3 depletion.

Arp3-depleted cells had major difficulties to conquer space around them (Figure 6B). As frequent and extended pausing should have equally affected the results on migration speeds, unproductive directional changes most likely caused these observations. And indeed, assessment of directional migration, which can be done in a fashion unbiased by cell speed (Dang and Gautreau, 2018), was also consistently and dramatically impaired upon Arp3 depletion in all three cell lines (Figure 6C).
So Arp2/3 complex activity in our fibroblasts appears more important for maintaining directionality of migration than the sole rate of motility, consistent with the finding that the Arp2/3 inhibitor Arpin promotes cell turning (Dang et al., 2013; Dang and Gautreau, 2018). This also fits the observation of compromised wound healing efficiency observed above (Figure 5 and Supplementary Video 2). However, to what extent does the observed inability to maintain directionality of migration 


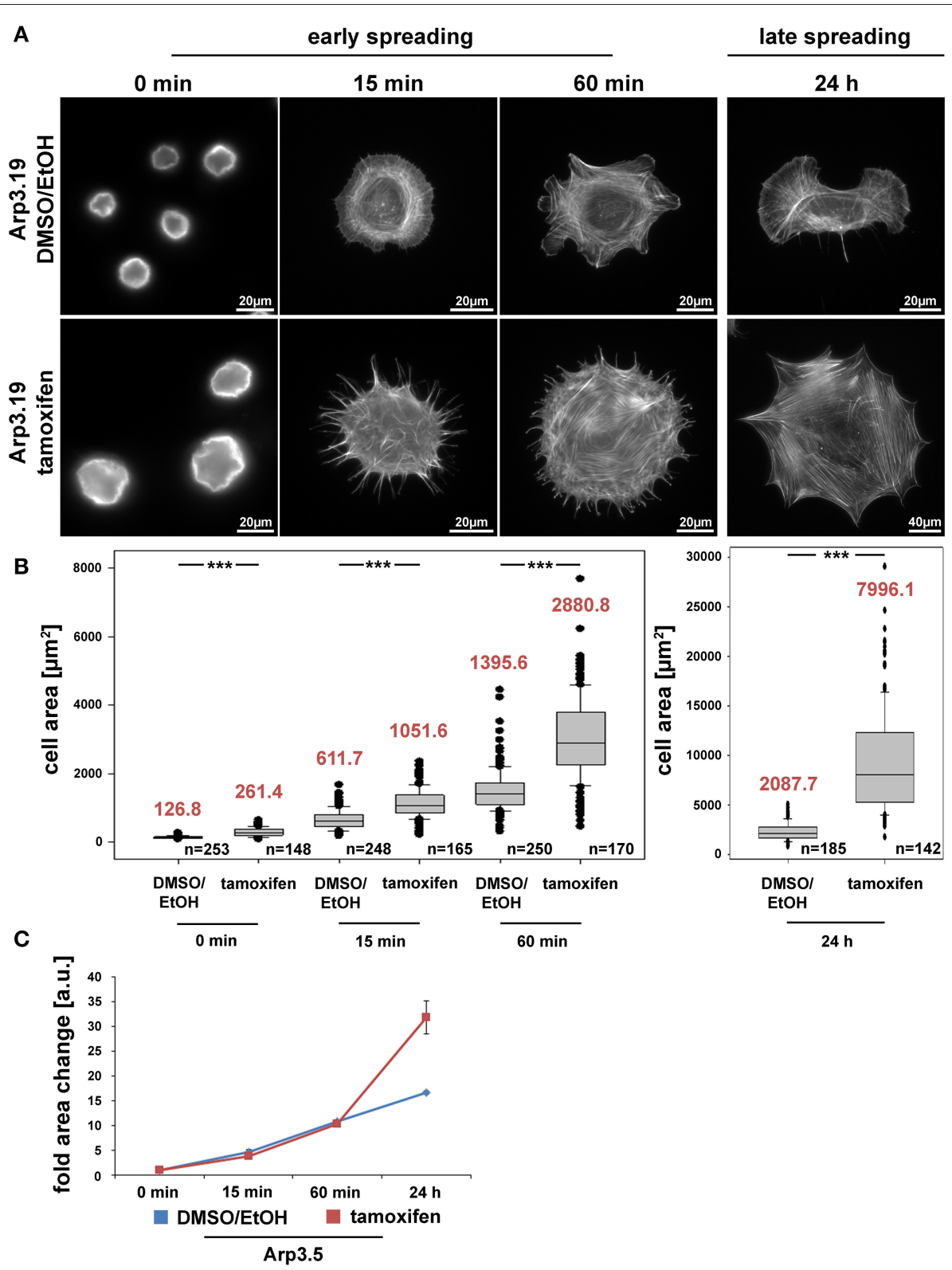

FIGURE 4 | The Arp2/3 complex is not required for cell spreading. (A) Phalloidin stainings of Actr $3^{\text {fl/fll }}$ cells (clone Arp3.19) with or without tamoxifen treatment (96 h) and subjected to cell spreading for different time points (0, 15, $60 \mathrm{~min}$, and $24 \mathrm{~h}$ ). Except for the 0-min time point, for which poly-L-lysine was used (see Methods), cells were allowed to spread on $25 \mathrm{\mu g} / \mathrm{ml}$ fibronectin. (B) Box and whiskers plots displaying quantification of spreading area using images as shown in (A). Boxes include $50 \%(25-75 \%)$ and whiskers $80 \%(10-90 \%)$ of all measurements. Outliers are shown as dots. Median values are given in red. $n=$ total number of cells analyzed from three independent experiments. Differences in average cell area of control and tamoxifen-treated cells at different time points of spreading were

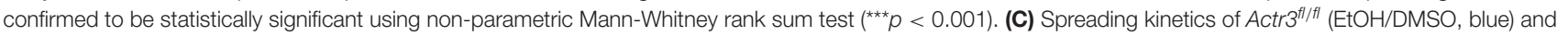
tamoxifen-treated cells (red) reported as fold change after normalization to cell size at time point 0 . Data taken from (B). Data are arithmetic means and error bars represent SEMs. Note that Arp3 knockout cells spread with kinetics highly similar to corresponding control cells, but adopt a much larger area $24 \mathrm{~h}$ after seeding, due perhaps to continuous increase in cell size effected by Arp3 removal.

in random migration assays affect chemotactic performance in our cell lines both with and without Arp2/3 complex? For this, we probed for chemotactic migration toward HGF $(100 \mathrm{ng} / \mathrm{ml})$ and $2.5 \%$ serum, as used previously for Rac1deficient fibroblasts (Steffen et al., 2013). In this previous study, we concluded that cells lacking this prominent upstream
GTPase, which is considered essential for WRC- and Arp2/3 complex-driven, lamellipodial actin assembly, are incapable of chemotactic migration (Steffen et al., 2013). Thus, it was reasonable to assume that similar results would be obtained with its prominent, direct downstream effector of actin nucleation, the Arp $2 / 3$ complex, in spite of clearly conflicting data in 


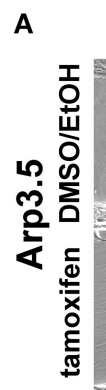

$\mathbf{O h}$
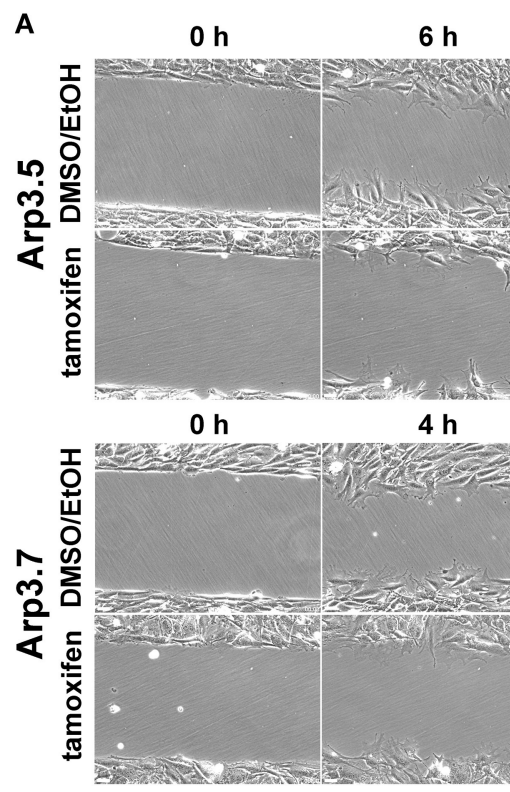

$\mathrm{Oh}$
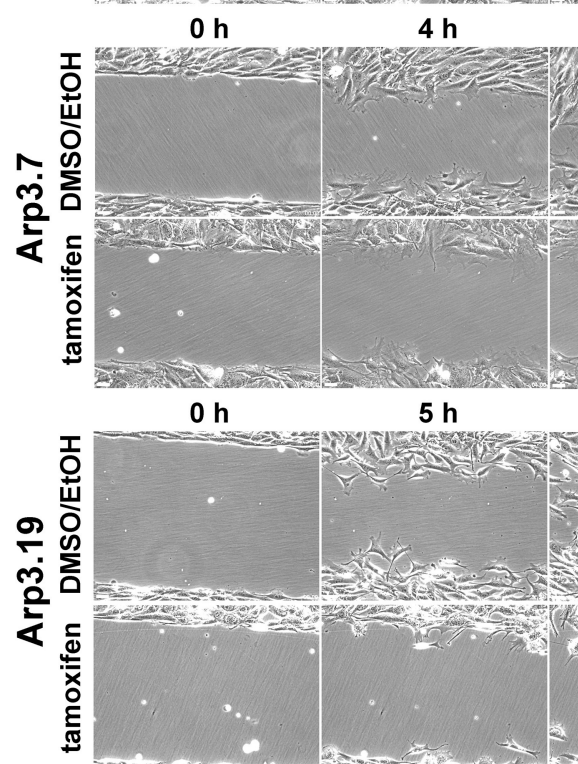

$\mathbf{O} \mathbf{h}$

$5 \mathrm{~h}$

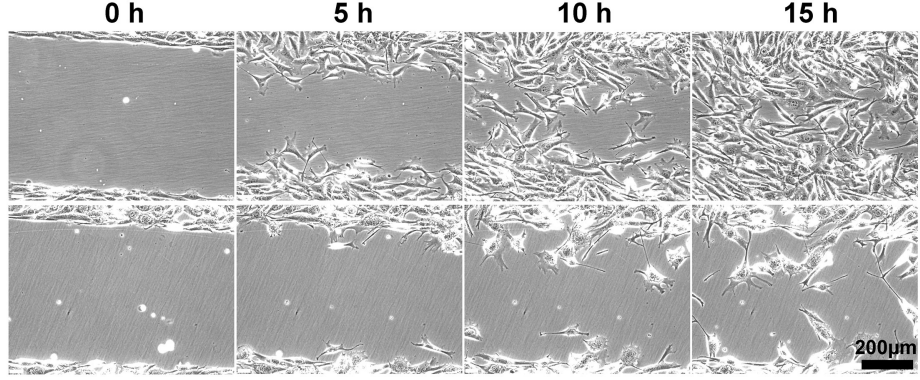

$12 \mathrm{~h}$

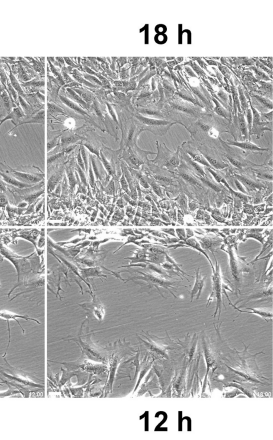

$8 \mathrm{~h}$

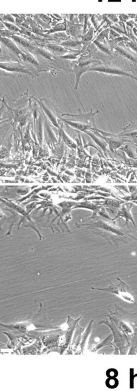

B

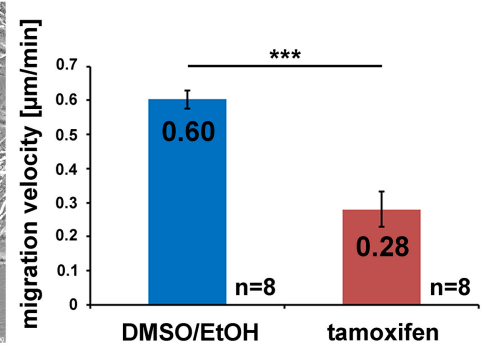

FIGURE 5 | Acute Arp2/3 complex removal reduces wound closure capacity. (A) Selected frames from wound healing, time-lapse microscopy (24 h) of control- and tamoxifen-treated Actr ${ }^{f / f l}$ clones. Wounds closed on average after roughly $18 \mathrm{~h}$ in controls, but failed to do so in Arp3 null cells. (B) Quantification of collective migration velocity during wound closure utilizing movies as displayed in (A). Bar charts show arithmetic means and SEMs. $n=$ number of movies analyzed, generated from three independent experiments. Differences in wound closure speed between individual control- and tamoxifen-treated clones were confirmed to be statistically significant using two-sided two-sample $t$-test $\left({ }^{\star \star \star} p<0.001\right)$.

this respect with alternative, Arp2/3 complex-depleted fibroblast cell models (Wu et al., 2012, 2013). Importantly, however, the findings described here largely confirm aforementioned data published by the Bear-lab, as the principal capability to chemotax was still observed upon Arp3 depletion (Figure 7, clone 19). In fact, the forward migration index (FMI) was only moderately reduced and the defect more severe if considering overall chemotactic migration rate (Figure 7D), perhaps again caused by the difficulty to maintain directionality of migration. In any case, our data shed more light on this long-standing controversy on the precise function of Arp2/3 complex in chemotaxis, as we do find a partial contribution of the presence of Arp2/3 complex in such assays, even though it does not appear to be obligatory, as concluded from other previous data (Suraneni et al., 2012, 2015). Virtually identical results were obtained in independent experiments using clones 5 (Supplementary Figure 10) and 7 (Supplementary Figure 11).

\section{Defective Directional Migration Coincides With Altered Focal Adhesion Patterns}

Previous studies have suggested effects of Arp2/3 complexdepletion on certain focal adhesion features, in particular concerning their alignment with each other, which is promoted by functional Arp2/3 complex and the presence of lamellipodia (Wu et al., 2012). Apart from this apparent phenotype (Figure 8A), we saw a significant increase in overall adhesion numbers per cell (Figure 8B), but this was reverted to the opposite if the large increase in cell area effected by acute Arp2/3 complex removal was considered (Figure 8C). Finally, we also found a significant increase in average sizes of individual focal adhesions (Figure 8D). It is commonly agreed upon that proper focal adhesion turnover is crucial for effective migration. Thus, we hypothesize that the unproductive directional changes and resulting defects in the efficiency of directional migration observed above may well be due to a combination of increased cell size and reduced numbers but increased sizes of individual 

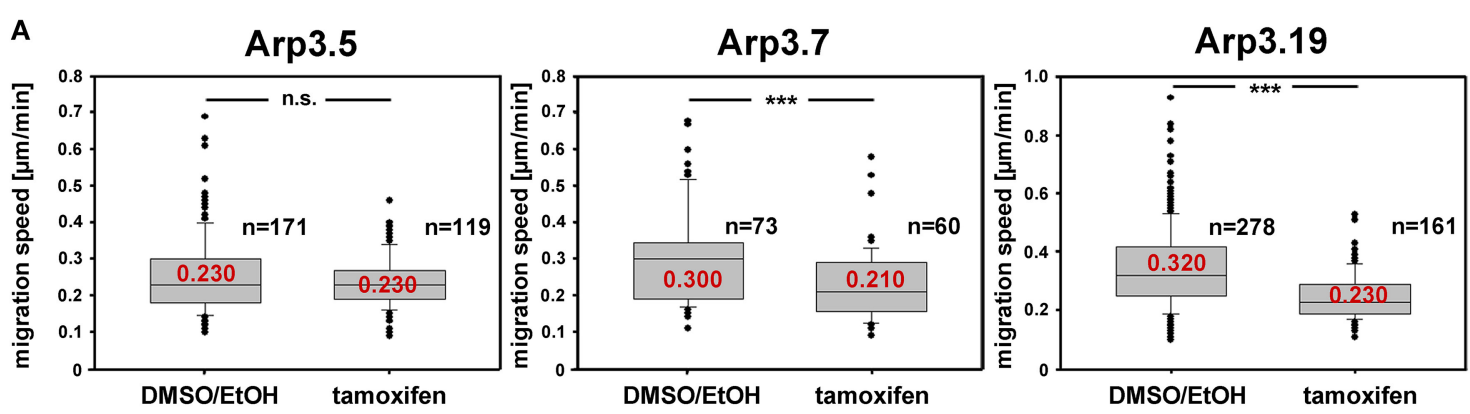

B

Arp3.5

Arp3.7
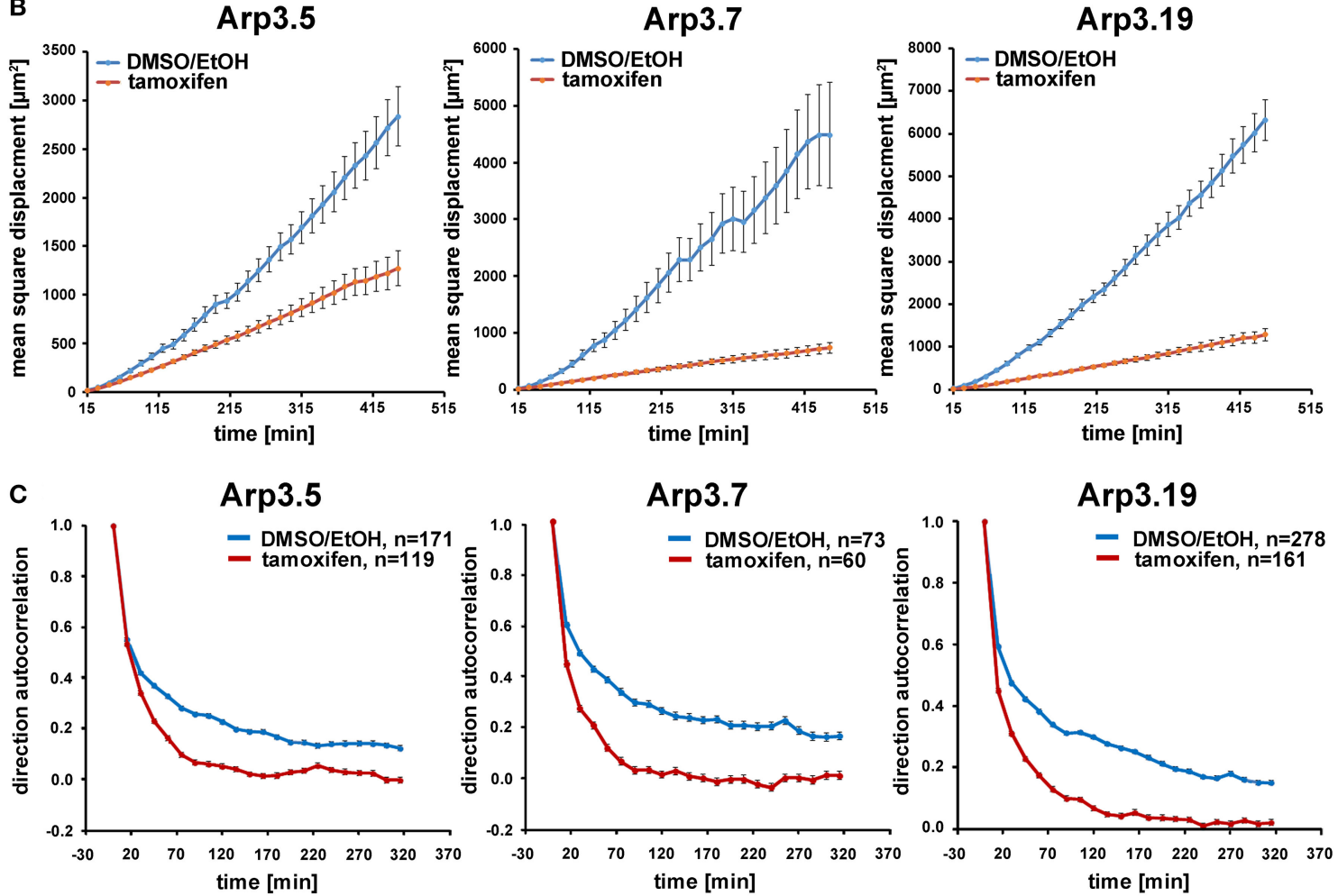

FIGURE 6 | Arp2/3 complex depletion has little effect on the speed of random cell migration, but diminishes efficiency by compromising migration directionality. (A) Random migration speed of Actr $3^{f / f t}$ clones (Arp3.5, Arp3.7, and Arp3.19, as indicated) with or without treatment with tamoxifen causing acute gene disruption. Data obtained from time-lapse microscopy $(20 \mathrm{~h}$ ) followed by manual tracking of individual cells (for individual cell tracks see Supplementary Figure 9). Box and whiskers plots were as described for Figure 4B, and data derived from three independent experiments. $n=$ total number of cells analyzed. Non-parametric Mann-Whitney rank sum test was used for statistical comparison of respective groups ( $\left.{ }^{\star \star}{ }^{*} p<0.001\right)$. (B) Mean square displacement analysis of the same data as shown in (A), revealing the strong decrease in migration efficiency upon Arp3 depletion in spite of the limited impact on speed. (C) Analysis of directional migration utilizing trajectories from (A) and shown in Supplementary Figure 9. An autocorrelation of 1 corresponds to strictly directed movement whereas an autocorrelation of 0 corresponds to pure random walk. Note the rapid and consistent loss of directional migration upon Arp2/3 complex removal (tamoxifen) in these experimental conditions in all three cell clones. Data are displayed as direction autocorrelation curves with error bars representing standard errors of means.

focal adhesions. These features may well-interfere with the flexibility of focal adhesion dynamics and turnover normally required to promote the maintenance of directionality. Although hitherto restricted to analyses in $2 \mathrm{D}$ assays here, we speculate that such defects will even be potentiated in more complex, 3D environments, likely increasing the need for rapidly adapting to environmental variability. Such a scenario is already consistent with the crucial function of Arp3 in populating the mouse skin with melanocytes, as established recently (Papalazarou et al., 2020).

\section{Acute Arp3 Loss of Function Increases Expression of FMNL Subfamily Formins That Operate in Filopodia Formation}

Our previous studies have established both Arp $2 / 3$ complex and FMNL subfamily formins to co-operate in the efficiency of lamellipodia formation (Block et al., 2012; Kage et al., 2017). However, in the absence of lamellipodia, such as upon Rac1 deletion, FMNL formins can only trigger filopodia but not rescue lamellipodia formation (Kage et al., 2017). In 


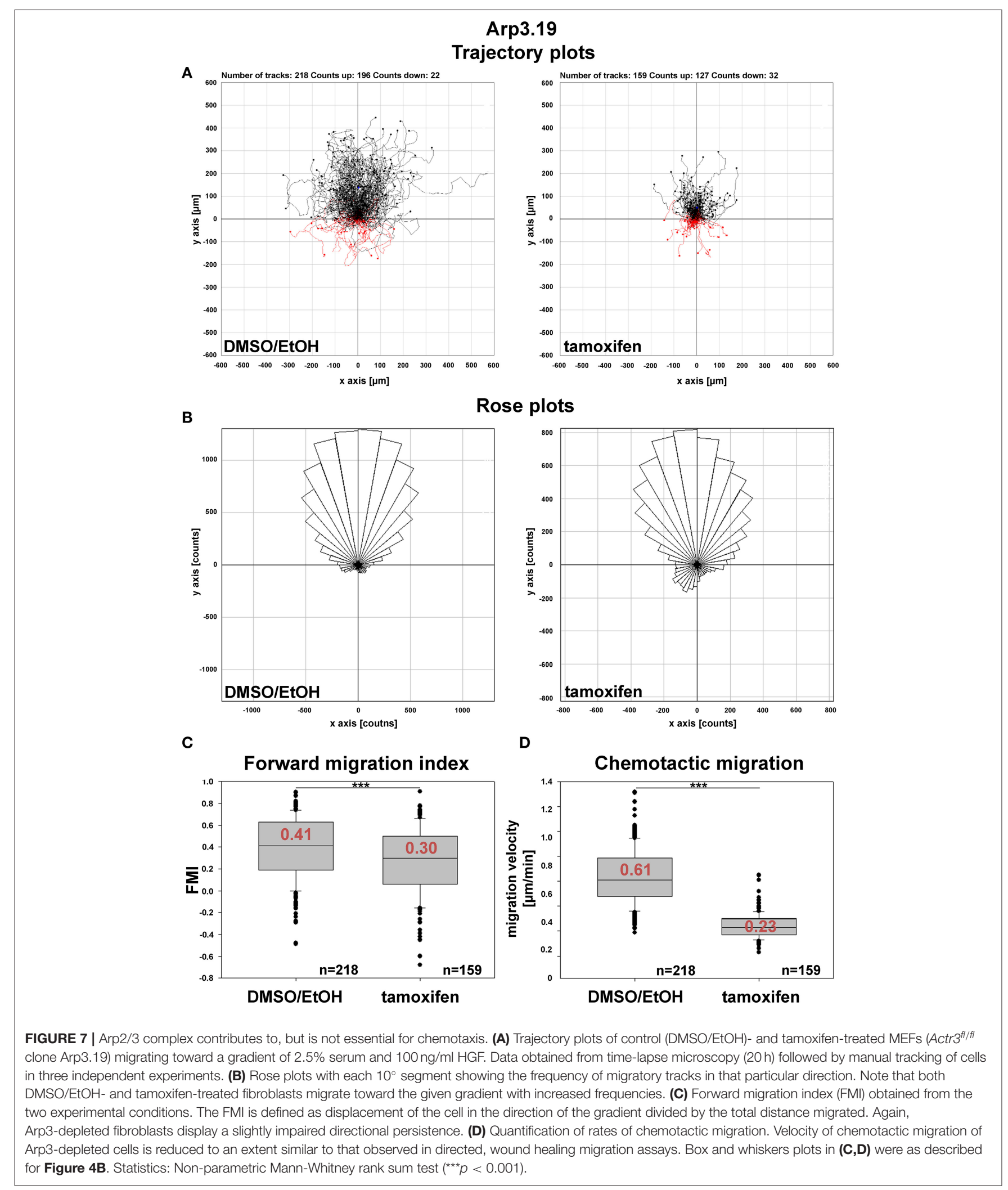

these experiments, active FMNL3, which can promote actin filament nucleation independent of Arp2/3 complex in vitro, prominently induced the formation of filopodia with club-shaped appearance (Kage et al., 2017), a feature that had previously also been attributed to active actin filament nucleation in cells (Yang et al., 2007; Block et al., 2008). Interestingly, FMNL3 


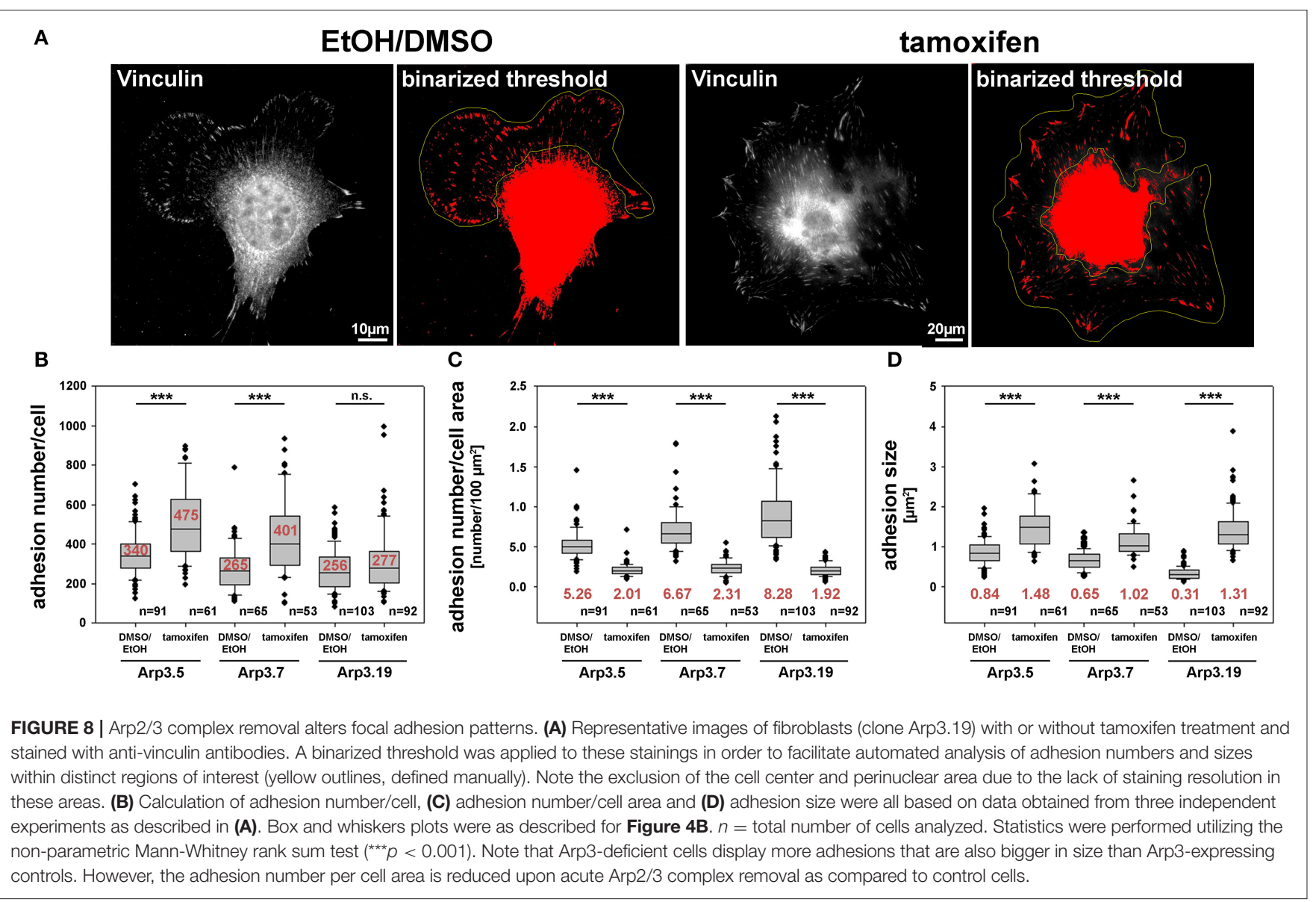

knockdown was recently also found to suppress filopodia formation in U2OS osteocarcoma cells (Young et al., 2018). In a screen for potentially compensatory upregulation of prominent actin binding proteins, both FMNL2 and FMNL3 appeared significantly enhanced in expression upon induced Arp3 suppression (Figures 9A,B). Since tamoxifen-induced Arp3 depletion clearly increased cell edge complexity, with numerous, small, concave-shaped regions at the cell periphery (Figure 2A and Supplementary Figure 12A), and stronglyincreased filopodia numbers (Supplementary Figure 12B), we asked whether RNAi-mediated depletion of induced FMNL formins would revert these effects. First, we confirmed the efficiency of combined FMNL2 and-3 knockdown with and without tamoxifen treatment (Figure 9C). Knockdown efficiencies appeared admittedly moderate in these conditions. However, quantifications revealed a relative suppression of expression by 56.5 and $22.7 \%$ for FMNL2 and-3, respectively, in control cells, and by 45.3 and $36.6 \%$ upon Arp3-KO. For filopodia quantification, we chose conditions in which filopodia numbers were maximal, which was the case during spreading (Figures 9D,E). Interestingly, filopodia numbers seen in the presence of Arp2/3 complex in these conditions, were not affected by FMNL2/3 knockdown (for quantification see Figure 9E), but the increase in filopodia numbers effected by Arp3 removal appeared abolished (Figure 9E). Significantly, FMNL2/3 RNAi also strongly interfered with the spreading increase induced by Arp3 removal in these experiments (Figure 9F), confirming the conclusion that filopodia can indeed promote this process (see above and Supplementary Video 1). We should emphasize that additional actin polymerases have previously been implicated in filopodia formation, foremost of all Ena/VASP family members (Dent et al., 2007), although they are certainly not obligatory for these structures (Damiano-Guercio et al., 2020). It is tempting to speculate that during spreading in the presence of Arp2/3 complex, Ena/VASP proteins are more relevant for the formation of the filopodia formed under these conditions than FMNL2/3 proteins. Interestingly, the Ena/VASP members VASP and Mena were also found to be upregulated in expression in a previous Arp2/3-KO model (Rotty et al., 2015), and we were able to confirm this for VASP upon induced Arp3 removal here (data not shown). Thus, the precise relative contributions of both FMNL formin and Ena/VASP families to filopodia formation in the presence or absence of Arp2/3 complex remains to be established in the future. Notwithstanding this, we show here that FMNL formin upregulation has clear effects on the changes of features of protrusion and cell morphology seen upon acute Arp3 removal (Figure 9). In spread cells of regular cultures, filopodia formation was virtually absent in 

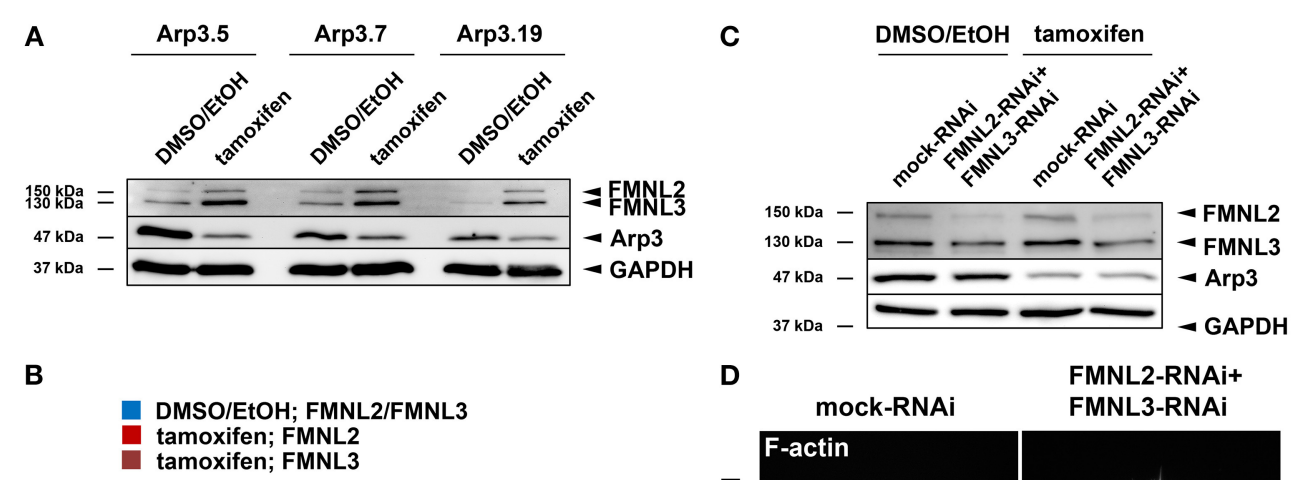

。
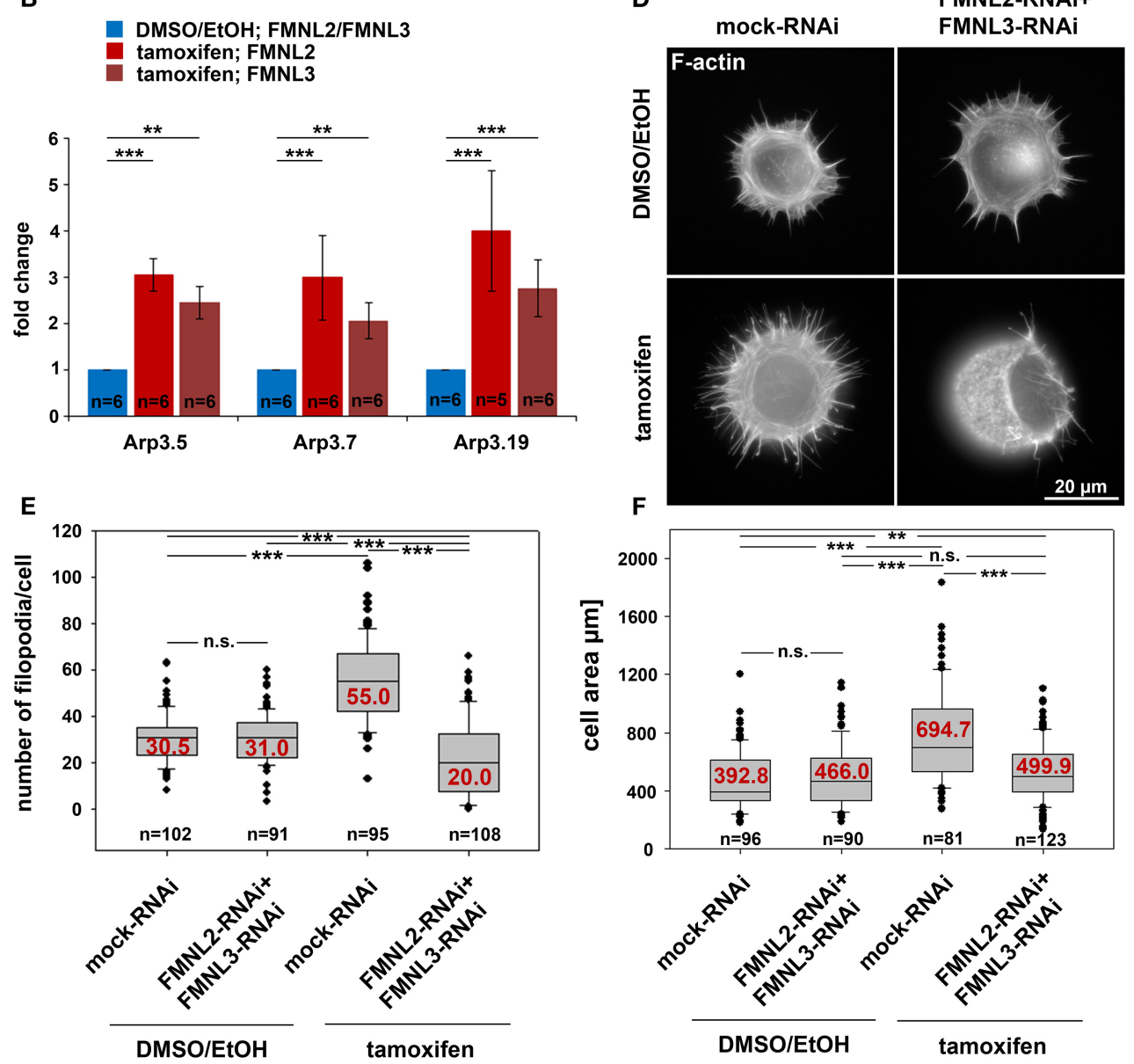

FIGURE 9 | Acute Arp3 removal up-regulates FMNL formin expression enhancing filopodia formation and cell spreading. (A) Representative Western blot of Arp3 as well as FMNL2 and FMNL3 protein levels in Actr3 ${ }^{f / f l}$ clones with and without tamoxifen treatment, as indicated. GAPDH served as loading control. (B) Quantification of FMNL2 and FMNL3 protein levels from Western blots as shown in (A). Bar charts display arithmetic means of FMNL2 and-3 protein levels normalized to GAPDH, with each FMNL variant (shades of red) being presented as fold-change relative to its respective control (blue). Error bars represent SEMs; data obtained from at least five independently generated cell extracts. Statistics were performed utilizing non-parametric Mann-Whitney rank sum test $\left({ }^{* \star *} p<0.001 ;{ }^{* \star}<0.01\right)$. Note the significant elevation of FMNL2 and-3 protein levels upon Arp3 suppression in all three clones. (C) Representative Western blot analysis of FMNL2 and FMNL3 protein amounts in detergent-soluble extracts from DMSO/EtOH- and tamoxifen-treated Actr3 $3^{f / f l}$ cells (clone Arp3.19) after co-transfection with RNAi plasmids individually downregulating FMNL2 and FMNL3 expression or mock-plasmid as control, as indicated. Note the moderate but consistent reduction of FMNL2 and-3 protein levels with and without tamoxifen-induced Arp3 depletion, as indicated (for quantification results see main text). (D) Representative phalloidin stainings of Actr $3^{f / f l}$ cells (clone Arp3.19) control or tamoxifen-treated, and combined with mock- or FMNL2/3-RNAi after 15 min of spreading on fibronectin-coated coverslips. (E,F) Quantification of filopodia formation (E) and spreading area (F) of cells as presented in (D); box and whiskers plots as described for Figure 4B. $n=$ total number of cells analyzed from three independent experiments. Statistics were performed utilizing non-parametric, Mann-Whitney rank sum test ${ }^{\star * \star *} p<0.001 ;{ }^{* \star}<0.01$; n.s. not significant). Note that Arp3 knockout is accompanied by a pronounced increase in filopodia numbers that are reduced even below Arp3-treatment control levels upon FMNL2/3 RNAi. In addition, the increase in tamoxifen-induced cell area upon 15 min of spreading is also suppressed significantly by FMNL2/3 knockdown.

the presence of Arp2/3-complex, but the increase effected by Arp3 removal was again almost entirely eliminated through additional FMNL2/3 knockdown (Supplementary Figure 12B).
Alongside with this, cell edge complexity was also severely reduced upon FMNL2/3 knockdown in Arp3-depleted cells, as evidenced by a strong reduction of the numbers of concave 


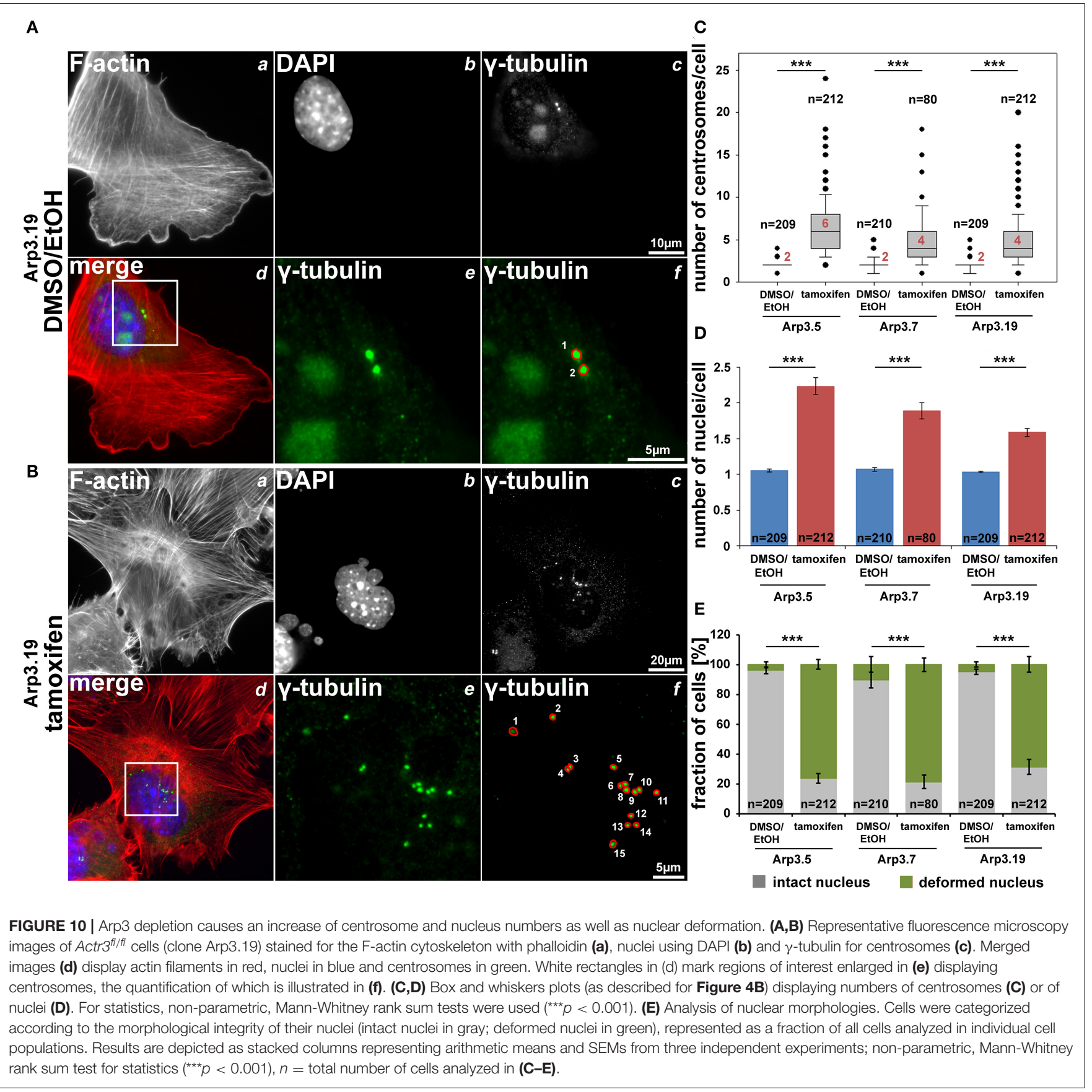

edges (Supplementary Figure 12C), which of course correlated with an increased size of remaining concave edges upon additional FMNL2/3 knockdown (Supplementary Figure 12D). Furthermore, the impact of FMNL2/3 knockdown on the Arp3KO-induced increase of cell area was much less pronounced in these spread cells (Supplementary Figure 12E) than during early spreading (compare with Figure 9F), which can well be explained by this parameter in already spread cells being mostly derived from the Arp3-KO-triggered increase in cell size.
Finally, spreading cells $(15 \mathrm{~min})$ suppressed in expression of both, Arp $2 / 3$ complex and FMNL2/3 displayed severe defects in overall cell shape and actin filament distribution at the cell periphery (Figure 9D bottom right), nicely illustrated by SIM superresolution imaging (Supplementary Figure 13 bottom right panel). Aside from the apparent, massive induction of small, bleb-like structures, both epifluorescence (Supplementary Figure 14A) and SIM imaging (Supplementary Figure 14B) of Arp3-KO/FMNL2/3 knockdown cells revealed the frequent formation of distinct, 
oval-shaped regions of very low F-actin content. These regions, likely mediating cell-substratum adhesion, did not coincide with the shape of the nucleus (Supplementary Figure 15). Most strikingly, however, 3D-projections of SIM imaging data of control (Supplementary Video 3) vs. Arp3/FMNL2/3depleted cells (for representative examples without and with nuclear staining see Supplementary Videos 4-6, respectively) clearly revealed a drastic change of overall cell shape upon interference with expression of both Arp3 and FMNL2/3 formins. Representatives of the latter treatment frequently adopted the shape of thick disks during spreading instead of the flat, cone-shaped cells observed in the control situation. The physics behind this phenomenon requires further, thorough investigation, including potential effects on stability and nature of the actin cortex in these conditions (Bovellan et al., 2014). Notwithstanding this, our data unequivocally show that the drastic changes in cell morphology effected by acute Arp3 removal, e.g., the observed increase of the numbers of filopodia or of concave regions formed along the cell edge, are to large extent mediated by the induced upregulation of FMNL2/3 formin expression.

\section{Acute Arp3 Removal Increases Centrosome and Nucleus Numbers and Causes Nuclear Deformation}

As described above, we have been unable to generate clones of immortalized fibroblast cell lines lacking Arp3. This suggested that expression of Arp3 was essential for cell division or perhaps cytokinesis. Indeed, Arp2/3 complex function has previously been linked to proper cell division and mitotic spindle formation (Plessner et al., 2019), perhaps through actin-dependent tuning of centrosomal microtubule nucleation (Farina et al., 2019), although it is already clear that Arp2/3 complex cannot be obligatory for cell division in all cell types and conditions (Graziano et al., 2019). Here we found that induced, acute Arp3 removal in fibroblasts causes dramatic increase in average centrosome numbers from roughly 2 to $>5$ on average in interphase cells (Figures 10A-C, Supplementary Figures 15, 16). Severe problems with cytokinesis were also suggested by the increase of average nuclei numbers observed in all three cell clones after acute Arp3 reduction (Figure 10D). Finally, many nuclei appeared swollen, fragmented or deformed, with frequent "budshapelike" protrusions, which were very rarely seen in control DMSO/EtOH-treated cells. In contrast, the percentage of cells displaying at least one deformed nucleus was increased to roughly $70-80 \%$ upon tamoxifen treatment, dependent on the clone treated (Figures 10B,E). To what extent Arp2/3-dependent actin assembly might contribute to nuclear envelope rupture as previously established in starfish oocytes (Wesolowska et al., 2020) and as pre-requisite for cells to enter metaphase of mitosis, remains to be established in future studies. Clearly, a potential, specific function in nuclear envelope rupture and breakdown would at least be consistent with the deformed nucleus phenotype observed here (Figure 10B and
Supplementary Figures 15B, 16B). However, future research is needed to dissect whether the obligatory function in cell division and growth observed for the Actr3 gene studied here reflects a cell type- or condition-dependent function in these processes for Arp2/3 complex-dependent actin remodeling or a more general, Arp2/3 complex-independent function of the Actr3 gene in all cells and tissues (Vauti et al., 2007).

\section{DATA AVAILABILITY STATEMENT}

The raw data supporting the conclusions of this article will be made available by the authors, without undue reservation.

\section{AUTHOR CONTRIBUTIONS}

VD performed all experiments except for the wound healing assays, which were done by SK. FK helped with SIM data generation and cloned FMNL knockdown constructs. IL generated the mouse model harboring loxP site-flanked Actr3 alleles, established and provided spontaneously immortalized Actr $3^{f / f l}$ cell lines and performed initial experiments. GD, AS, and TS provided essential reagents and supported data analysis and interpretation. FV aided construct generation for homologous recombination and all experiments involving generation of the mouse mutant. H-HA conceptualized the Arp3 knockout study, supervised all stages of the project, and helped developing the cellular work. KR supervised all cell experiments, interpreted data and wrote the paper. All authors contributed to data interpretation and development of conclusions, and revised, read and approved the manuscript text.

\section{FUNDING}

This work was supported in part by the Deutsche Forschungsgemeinschaft (DFG), grants Ar115/6-2 (to H-HA), RO2414/3-2 and RO2414/5-1 (to KR) as well as the Seventh Framework Programme grant FORCEFULACTIN of the European Research Council (to Marie-France Carlier and KR).

\section{ACKNOWLEDGMENTS}

We thank Sophia Leschik for experimental help during early stages of the Project, Pierre Chambon, Laura Machesky and Giorgio Scita for reagents, Lothar Gröbe (HZI Braunschweig) for cell sorting, Magdalena Mietkowska for sharing unpublished data, and Gerd Landsberg (University of Bonn) and Brigitte Denker (HZI Braunschweig) for excellent technical assistance.

\section{SUPPLEMENTARY MATERIAL}

The Supplementary Material for this article can be found online at: https://www.frontiersin.org/articles/10.3389/fcell.2021. 634708/full\#supplementary-material 


\section{REFERENCES}

Abella, J. V., Galloni, C., Pernier, J., Barry, D. J., Kjaer, S., Carlier, M. F., et al. (2016). Isoform diversity in the Arp2/3 complex determines actin filament dynamics. Nat. Cell Biol. 18, 76-86. doi: 10.1038/ncb3286

Akamatsu, M., Vasan, R., Serwas, D., Ferrin, M. A., Rangamani, P., and Drubin, D. G. (2020). Principles of self-organization and load adaptation by the actin cytoskeleton during clathrin-mediated endocytosis. Elife 9:49840. doi: 10.7554/eLife.49840

Alekhina, O., Burstein, E., and Billadeau, D. D. (2017). Cellular functions of WASP family proteins at a glance. J. Cell Sci. 130, 2235-2241. doi: 10.1242/jcs.199570

Block, J., Breitsprecher, D., Kuhn, S., Winterhoff, M., Kage, F., Geffers, R., et al. (2012). FMNL2 drives actin-based protrusion and migration downstream of Cdc42. Curr. Biol. 22, 1005-1012. doi: 10.1016/j.cub.2012.03.064

Block, J., Stradal, T. E., Hanisch, J., Geffers, R., Kostler, S. A., Urban, E., et al. (2008). Filopodia formation induced by active mDia2/Drf3. J. Microsc. 231, 506-517. doi: 10.1111/j.1365-2818.2008.02063.x

Bovellan, M., Romeo, Y., Biro, M., Boden, A., Chugh, P., Yonis, A., et al. (2014). Cellular control of cortical actin nucleation. Curr. Biol. 24, 1628-1635. doi: 10.1016/j.cub.2014.05.069

Breitsprecher, D., Jaiswal, R., Bombardier, J. P., Gould, C. J., Gelles, J., and Goode, B. L. (2012). Rocket launcher mechanism of collaborative actin assembly defined by single-molecule imaging. Science 336, 1164-1168. doi: $10.1126 /$ science. 1218062

Campellone, K. G., and Welch, M. D. (2010). A nucleator arms race: cellular control of actin assembly. Nat. Rev. Mol. Cell Biol. 11, 237-251. doi: $10.1038 / \mathrm{nrm} 2867$

Chesarone, M. A., and Goode, B. L. (2009). Actin nucleation and elongation factors: mechanisms and interplay. Curr. Opin Cell Biol. 21, 28-37. doi: 10.1016/j.ceb.2008.12.001

Chorev, D. S., Moscovitz, O., Geiger, B., and Sharon, M. (2014). Regulation of focal adhesion formation by a vinculin-Arp2/3 hybrid complex. Nat. Commun. 5:3758. doi: $10.1038 /$ ncomms4758

Damiano-Guercio, J., Kurzawa, L., Mueller, J., Dimchev, G., Schaks, M., Nemethova, M., et al. (2020). Loss of Ena/VASP interferes with lamellipodium architecture, motility and integrin-dependent adhesion. Elife 9:e55351. doi: 10.7554/eLife.55351.sa2

Dang, I., and Gautreau, A. (2018). Random migration assays of mammalian cells and quantitative analyses of single cell trajectories. Methods Mol. Biol. 1749, 1-9. doi: 10.1007/978-1-4939-7701-7_1

Dang, I., Gorelik, R., Sousa-Blin, C., Derivery, E., Guerin, C., Linkner, J., et al. (2013). Inhibitory signalling to the Arp $2 / 3$ complex steers cell migration. Nature 503, 281-284. doi: 10.1038/nature12611

Dent, E. W., Kwiatkowski, A. V., Mebane, L. M., Philippar, U., Barzik, M., Rubinson, D. A., et al. (2007). Filopodia are required for cortical neurite initiation. Nat. Cell Biol. 9, 1347-1359. doi: 10.1038/ncb1654

Di Nardo, A., Cicchetti, G., Falet, H., Hartwig, J. H., Stossel, T. P., and Kwiatkowski, D. J. (2005). Arp2/3 complex-deficient mouse fibroblasts are viable and have normal leading-edge actin structure and function. Proc. Natl. Acad. Sci. U.S.A. 102, 16263-16268. doi: 10.1073/pnas.0508228102

Dimchev, G., and Rottner, K. (2018). Micromanipulation techniques allowing analysis of morphogenetic dynamics and turnover of cytoskeletal regulators. J. Vis. Exp. 12:135. doi: 10.3791/57643

Dominguez, R. (2010). Structural insights into de novo actin polymerization. Curr. Opin Struct. Biol. 20, 217-225. doi: 10.1016/j.sbi.2009.12.012

Farina, F., Ramkumar, N., Brown, L., Samandar Eweis, D., Anstatt, J., Waring, T., et al. (2019). Local actin nucleation tunes centrosomal microtubule nucleation during passage through mitosis. EMBO J. 38:e99843. doi: $10.15252 / \mathrm{embj} .201899843$

Feil, R., Wagner, J., Metzger, D., and Chambon, P. (1997). Regulation of Cre recombinase activity by mutated estrogen receptor ligand-binding domains. Biochem. Biophys. Res. Commun. 237, 752-757. doi: 10.1006/bbrc.1997.7124

Galloni, C., Carra, D., Abella, J. V., Kjaer, S., Singaravelu, P., Barry, D. J., et al. (2020). MICAL2 acts through Arp3B isoform-specific Arp2/3 complexes to destabilize branched actin networks. bioRxiv preprint. 1-27. doi: 10.1101/2020.09.21.306522

Goley, E. D., and Welch, M. D. (2006). The ARP2/3 complex: an actin nucleator comes of age. Nat. Rev. Mol. Cell Biol. 7, 713-726. doi: 10.1038/nrm2026
Gorelik, R., and Gautreau, A. (2014). Quantitative and unbiased analysis of directional persistence in cell migration. Nat. Protoc. 9, 1931-1943. doi: 10.1038/nprot.2014.131

Graziano, B. R., Town, J. P., Sitarska, E., Nagy, T. L., Fosnaric, M., Penic, S., et al. (2019). Cell confinement reveals a branched-actin independent circuit for neutrophil polarity. PLoS Biol. 17:e3000457. doi: 10.1371/journal.pbio.3000457

Hufner, K., Higgs, H. N., Pollard, T. D., Jacobi, C., Aepfelbacher, M., and Linder, S. (2001). The verprolin-like central (vc) region of Wiskott-Aldrich syndrome protein induces Arp2/3 complex-dependent actin nucleation. J. Biol. Chem. 276, 35761-35767. doi: 10.1074/jbc.M106520200

Jay, P., Berge-Lefranc, J. L., Massacrier, A., Roessler, E., Wallis, D., Muenke, M., et al. (2000). ARP3beta, the gene encoding a new human actin-related protein, is alternatively spliced and predominantly expressed in brain neuronal cells. Eur. J. Biochem. 267, 2921-2928. doi: 10.1046/j.1432-1327.2000.01306.x

Kadzik, R. S., Homa, K. E., and Kovar, D. R. (2020). F-actin cytoskeleton network self-organization through competition and cooperation. Annu. Rev. Cell Dev. Biol. 36, 35-60. doi: 10.1146/annurev-cellbio-032320-094706

Kage, F., Winterhoff, M., Dimchev, V., Mueller, J., Thalheim, T., Freise, A., et al. (2017). FMNL formins boost lamellipodial force generation. Nat. Commun. 8:14832. doi: $10.1038 /$ ncomms 14832

Kerkhoff, E. (2011). Actin dynamics at intracellular membranes: the Spir/formin nucleator complex. Eur. J. Cell Biol. 90, 922-925. doi: 10.1016/j.ejcb.2010.10.011

King, S. J., Asokan, S. B., Haynes, E. M., Zimmerman, S. P., Rotty, J. D., Alb, J. G. Jr., et al. (2016). Lamellipodia are crucial for haptotactic sensing and response. J. Cell Sci. 129, 2329-2342. doi: 10.1242/jcs. 184507

Koestler, S. A., Steffen, A., Nemethova, M., Winterhoff, M., Luo, N., Holleboom, J. M., et al. (2013). Arp $2 / 3$ complex is essential for actin network treadmilling as well as for targeting of capping protein and cofilin. Mol. Biol. Cell 24, 2861-2875. doi: 10.1091/mbc.e12-12-0857

Krause, M., and Gautreau, A. (2014). Steering cell migration: lamellipodium dynamics and the regulation of directional persistence. Nat. Rev. Mol. Cell Biol. 15, 577-590. doi: $10.1038 / \mathrm{nrm} 3861$

Lahmann, I. (2011). Konditionelle Mutagenese des Arp3-Gens in der Maus: Untersuchungen zur Funktion in der Skelettmuskelentwicklung in vivo und in embryonalen Fibroblasten in vitro. Dissertation, Technical University of Braunschweig.

Lai, F. P., Szczodrak, M., Block, J., Faix, J., Breitsprecher, D., Mannherz, H. G., et al. (2008). Arp2/3 complex interactions and actin network turnover in lamellipodia. $E M B O ~ J .27,982-992$. doi: 10.1038/emboj.2008.34

Leithner, A., Eichner, A., Muller, J., Reversat, A., Brown, M., Schwarz, J., et al. (2016). Diversified actin protrusions promote environmental exploration but are dispensable for locomotion of leukocytes. Nat. Cell Biol. 18, 1253-1259. doi: $10.1038 / \mathrm{ncb} 3426$

Machesky, L. M., and Insall, R. H. (1998). Scarl and the related Wiskott-Aldrich syndrome protein, WASP, regulate the actin cytoskeleton through the Arp2/3 complex. Curr. Biol. 8, 1347-1356. doi: 10.1016/S0960-9822(98)00015-3

Metzger, D., and Chambon, P. (2001). Site- and time-specific gene targeting in the mouse. Methods 24, 71-80. doi: 10.1006/meth.2001.1159

Molinie, N., and Gautreau, A. (2018). The Arp2/3 regulatory system and its deregulation in cancer. Physiol. Rev. 98, 215-238. doi: 10.1152/physrev.00006.2017

Montaville, P., Jegou, A., Pernier, J., Compper, C., Guichard, B., Mogessie, B., et al. (2014). Spire and Formin 2 synergize and antagonize in regulating actin assembly in meiosis by a ping-pong mechanism. PLoS Biol. 12:e1001795. doi: 10.1371/journal.pbio.1001795

Mueller, J., Szep, G., Nemethova, M., De Vries, I., Lieber, A. D., Winkler, C., et al. (2017). Load adaptation of lamellipodial actin networks. Cell 171:e116. doi: 10.1016/j.cell.2017.07.051

Nicholson-Dykstra, S. M., and Higgs, H. N. (2008). Arp2 depletion inhibits sheetlike protrusions but not linear protrusions of fibroblasts and lymphocytes. Cell Motil Cytoskeleton 65, 904-922. doi: 10.1002/cm.20312

Nolen, B. J., Tomasevic, N., Russell, A., Pierce, D. W., Jia, Z., Mccormick, C. D., et al. (2009). Characterization of two classes of small molecule inhibitors of Arp2/3 complex. Nature 460, 1031-1034. doi: 10.1038/nature08231

Olazabal, I. M., Caron, E., May, R. C., Schilling, K., Knecht, D. A., and Machesky, L. M. (2002). Rho-kinase and myosin-II control phagocytic cup formation during CR, but not FcgammaR, phagocytosis. Curr. Biol. 12, 1413-1418. doi: $10.1016 /$ S0960-9822(02)01069-2 
Papalazarou, V., and Machesky, L. M. (2020). The cell pushes back: the Arp2/3 complex is a key orchestrator of cellular responses to environmental forces. Curr. Opin Cell Biol. 68, 37-44. doi: 10.1016/j.ceb.2020.08.012

Papalazarou, V., Swaminathan, K., Jaber-Hijazi, F., Spence, H., Lahmann, I., Nixon, C., et al. (2020). The Arp2/3 complex is critical for colonisation of the mouse skin by melanoblasts. Development 147:dev194555. doi: 10.1242/dev.194555

Pizarro-Cerda, J., Chorev, D. S., Geiger, B., and Cossart, P. (2017). The diverse family of Arp2/3 complexes. Trends Cell Biol. 27, 93-100. doi: $10.1016 /$ j.tcb.2016.08.001

Plessner, M., Knerr, J., and Grosse, R. (2019). Centrosomal actin assembly is required for proper mitotic spindle formation and chromosome congression. Science 15, 274-281. doi: 10.1016/j.isci.2019.04.022

Qualmann, B., and Kessels, M. M. (2009). New players in actin polymerizationWH2-domain-containing actin nucleators. Trends Cell Biol. 19, 276-285. doi: 10.1016/j.tcb.2009.03.004

Rogers, S. L., Wiedemann, U., Stuurman, N., and Vale, R. D. (2003). Molecular requirements for actin-based lamella formation in Drosophila S2 cells. J. Cell Biol. 162, 1079-1088. doi: 10.1083/jcb.200303023

Rottner, K., Faix, J., Bogdan, S., Linder, S., and Kerkhoff, E. (2017). Actin assembly mechanisms at a glance. J. Cell Sci. 130, 3427-3435. doi: 10.1242/jcs.206433

Rottner, K., and Schaks, M. (2019). Assembling actin filaments for protrusion. Curr. Opin Cell Biol. 56, 53-63. doi: 10.1016/j.ceb.2018.09.004

Rotty, J. D., Wu, C., and Bear, J. E. (2013). New insights into the regulation and cellular functions of the ARP2/3 complex. Nat. Rev. Mol. Cell Biol. 14, 7-12. doi: $10.1038 / \mathrm{nrm} 3492$

Rotty, J. D., Wu, C., Haynes, E. M., Suarez, C., Winkelman, J. D., Johnson, H. E., et al. (2015). Profilin-1 serves as a gatekeeper for actin assembly by Arp2/3-dependent and -independent pathways. Dev. Cell 32, 54-67. doi: 10.1016/j.devcel.2014.10.026

Schaks, M., Giannone, G., and Rottner, K. (2019). Actin dynamics in cell migration. Essays Biochem. 63, 483-495. doi: 10.1042/EBC20190015

Schaks, M., Singh, S. P., Kage, F., Thomason, P., Klunemann, T., Steffen, A., et al. (2018). Distinct interaction sites of Rac GTPase with WAVE regulatory complex have non-redundant functions in vivo. Curr. Biol. 28:e3676. doi: 10.1016/j.cub.2018.10.002

Steffen, A., Faix, J., Resch, G. P., Linkner, J., Wehland, J., Small, J. V., et al. (2006). Filopodia formation in the absence of functional WAVE- and Arp2/3complexes. Mol. Biol. Cell 17, 2581-2591. doi: 10.1091/mbc.e05-11-1088

Steffen, A., Koestler, S. A., and Rottner, K. (2014). Requirements for and consequences of Rac-dependent protrusion. Eur. J. Cell Biol. 93, 184-193. doi: 10.1016/j.ejcb.2014.01.008

Steffen, A., Ladwein, M., Dimchev, G. A., Hein, A., Schwenkmezger, L., Arens, S., et al. (2013). Rac function is crucial for cell migration but is not required for spreading and focal adhesion formation. J. Cell Sci. 126, 4572-4588. doi: $10.1242 /$ jcs. 118232

Steffen, A., Rottner, K., Ehinger, J., Innocenti, M., Scita, G., Wehland, J., et al. (2004). Sra-1 and Nap1 link Rac to actin assembly driving lamellipodia formation. EMBO J. 23, 749-759. doi: 10.1038/sj.emboj. 7600084

Stradal, T., Courtney, K. D., Rottner, K., Hahne, P., Small, J. V., and Pendergast, A. M. (2001). The Abl interactor proteins localize to sites of actin polymerization at the tips of lamellipodia and filopodia. Curr. Biol. 11, 891-895. doi: 10.1016/S0960-9822(01)00239-1
Suarez, C., Carroll, R. T., Burke, T. A., Christensen, J. R., Bestul, A. J., Sees, J. A., et al. (2015). Profilin regulates F-actin network homeostasis by favoring formin over Arp2/3 complex. Dev. Cell 32, 43-53. doi: 10.1016/j.devcel.2014.10.027

Suraneni, P., Fogelson, B., Rubinstein, B., Noguera, P., Volkmann, N., Hanein, D., et al. (2015). A mechanism of leading-edge protrusion in the absence of Arp2/3 complex. Mol. Biol. Cell 26, 901-912. doi: 10.1091/mbc.E14-07-1250

Suraneni, P., Rubinstein, B., Unruh, J. R., Durnin, M., Hanein, D., and Li, R. (2012). The Arp2/3 complex is required for lamellipodia extension and directional fibroblast cell migration. J. Cell Biol. 197, 239-251. doi: 10.1083/jcb.201112113

Swaney, K. F., and Li, R. (2016). Function and regulation of the Arp2/3 complex during cell migration in diverse environments. Curr. Opin. Cell Biol. 42, 63-72. doi: 10.1016/j.ceb.2016.04.005

Vauti, F., Prochnow, B. R., Freese, E., Ramasamy, S. K., Ruiz, P., and Arnold, H. H. (2007). Arp3 is required during preimplantation development of the mouse embryo. FEBS Lett. 581, 5691-5697. doi: 10.1016/j.febslet.2007.11.031

Welch, M. D., and Way, M. (2013). Arp2/3-mediated actin-based motility: a tail of pathogen abuse. Cell Host Microbe 14, 242-255. doi: 10.1016/j.chom.2013.08.011

Wesolowska, N., Avilov, I., Machado, P., Geiss, C., Kondo, H., Mori, M., et al. (2020). Actin assembly ruptures the nuclear envelope by prying the lamina away from nuclear pores and nuclear membranes in starfish oocytes. Elife 9:e49774. doi: 10.7554/eLife.49774

Wu, C., Asokan, S. B., Berginski, M. E., Haynes, E. M., Sharpless, N. E., Griffith, J. D., et al. (2012). Arp2/3 is critical for lamellipodia and response to extracellular matrix cues but is dispensable for chemotaxis. Cell 148, 973-987. doi: 10.1016/j.cell.2011.12.034

Wu, C., Haynes, E. M., Asokan, S. B., Simon, J. M., Sharpless, N. E., Baldwin, A. S., et al. (2013). Loss of Arp2/3 induces an NF-kappaB-dependent, nonautonomous effect on chemotactic signaling. J. Cell Biol. 203, 907-916. doi: 10.1083/jcb.201306032

Yang, C., Czech, L., Gerboth, S., Kojima, S., Scita, G., and Svitkina, T. (2007). Novel roles of formin mDia2 in lamellipodia and filopodia formation in motile cells. PLoS Biol. 5:e317. doi: 10.1371/journal.pbio.0050317

Young, L. E., Latario, C. J., and Higgs, H. N. (2018). Roles for Ena/VASP proteins in FMNL3-mediated filopodial assembly. J. Cell Sci. 131:jcs220814. doi: $10.1242 /$ jcs. 220814

Conflict of Interest: The authors declare that the research was conducted in the absence of any commercial or financial relationships that could be construed as a potential conflict of interest.

Citation: Dimchev V, Lahmann I, Koestler SA, Kage F, Dimchev G, Steffen A, Stradal TEB, Vauti F, Arnold H-H and Rottner K (2021) Induced Arp2/3 Complex Depletion Increases FMNL2/3 Formin Expression and Filopodia Formation. Front. Cell Dev. Biol. 9:634708. doi: 10.3389/fcell.2021.634708

Copyright (C) 2021 Dimchev, Lahmann, Koestler, Kage, Dimchev, Steffen, Stradal, Vauti, Arnold and Rottner. This is an open-access article distributed under the terms of the Creative Commons Attribution License (CC BY). The use, distribution or reproduction in other forums is permitted, provided the original author(s) and the copyright owner(s) are credited and that the original publication in this journal is cited, in accordance with accepted academic practice. No use, distribution or reproduction is permitted which does not comply with these terms. 